\title{
Die Arten der Gattung Clinostomum Leidy.
}

\author{
Von \\ Prof. M. Braun, Königsberg Pr.
}

Hierzu Taf. 1 und 2.

Den vorläufigen Mittheilungen über Clinostomum LEIDY ${ }^{1}$ ) will ich eine ausführliche Beschreibung der einzelnen Arten folgen lassen, da kaum Aussicht vorhanden ist, dass mir selbst noch weiteres, unbearbeitetes Material zugehen wird und doch wohl mit der Unsicherheit auf diesem Gebiet ein Ende gemacht werden muss. Wenn die folgenden Ausführungen hierzu beitragen werden, so verdanke ich dies in erster Linie dem Umstand, dass mir auf meine Bitte von allen Seiten einschlägiges Material zuging; dies muss ich um so mehr hervorheben, als es sich um Raritäten, ja sogar um Unica handelte. Obgleich einzelne Objecte aus dem Anfang des Jahrhunderts stammen, war ihr Erhaltungszustand verhältnissmässig gut, freilich nicht so gut, um histologische Untersuchungen $\mathrm{zu}$ gestatten, die übrigens auch kaum zur Charakteristik der Arten nothwendig sind.

Welche Eigenthümlichkeiten besitzt die Gattung Clinostomum? Sie wurde bereits im Jahre 1856 von LeIDY ${ }^{2}$ ) mit den Species

1) Braun, M., Ueber Clinostomum Leidy, in: Zool. Anz., V. 22, 1899, p. 484 492. - Die Fascioliden-Gattung Clinostomum Leidr, in: Ctrbl. Bakt., V. 27, Abth. 1, 1900, p. 24-32. - Eine neue ClinostomumArt aus Ardea purpurea, in: Boll. Mus. Zool. Anat. comp. Torino, V. 14, No. 364, 1899.

2) Leidy, J., A synopsis of Entozoa, in: Proc. Acad. nat. Sc. Philadelphia, V. 8, p. $42-58$.

Die Diagnose der Gattung lautet: „Head shorter than and separated from the body by a lateral constriction opposite the ventral acetabulum,

Zool. Jahrb. XIV. Abth. f. Syst. 
C7. gracile und dubium aufgestellt, aber in der Literatur kaum beachtet, was durch die Dürftigkeit der Beschreibung beider Arten ${ }^{1}$ ) und die Seltenheit des Publicationsjournals wohl erklärlich erscheint. Daher konnte es nicht nur kommen, dass eine dem Clinostomum gracile sehr ähnliche Form ohne jede Bezugnahme auf dieses beschrieben wurde, sondern dass sie zum Vertreter einer anders genannten Gattung gemacht worden ist. Es handelt sich um Distomum reticulatum Looss ${ }^{2}$ ), das ebenso wenig wie Clinost. gracile im geschlechtsreifen Zustande vorlag, aber jeden Falls sehr viel besser von Looss beschrieben worden ist. Trotzdem nun R. LeUCKART ${ }^{3}$ ) auf die Uebereinstimmung der beiden in amerikanischen Fischen encystirt lebenden Arten hingewiesen hatte und dieser Hinweis auch Monticelli bekannt war, creirte dieser Autor für das Looss'sche Dist. reticulatum die Gattung Mesogonimus ${ }^{4}$ ). Aber wie wenig wird von den Eigenthümlichkeiten dieser Art für die Charakteristik der neuen Gattung benutzt! Nichts mehr als die Lage des Genitalporus und die etwas geringere Grösse des Mund- gegenüber dem Bauchsaugnapf.

Der Umstand, dass LEIDY bei der Beschreibung des Cl. gracile kein Wort über die Lage des Genitalporus sagt, kann die Aufstellung der Gattung Mesogonimus sehr wohl rechtfertigen, dagegen war es ein Fehler, die durch Looss genau genug beschriebenen und sonst bei

compressed semi-oval. Body compressed oval. Mouth anterior. Ventral acetabulum large, hemispherical, immersed within the commencement of the body and having a truncated conical aperture with the apex posterior. A terminal pore to the body."

1) Clin. gracile: „Head semi-ovoid, anteriorly obliquely truncated. Mouth transversaly oval, with a prominent margin and a second border, which is slightly emarginate below. Body compressed oblong, oval, convex above, concave below, obtuse posteriorly, acetabulum larger, immersed between the head and body. Length to 3 lines, breadth to 1 line." Esox sp. und Pomotis vulgaris.

Clin. dubium: „Head compressed oval, convex anteriorly; mouth minute, not bordered. Body compressed oblong oval. Ventral acetabulum immersed between the body and head. Length $2^{1 / 2}$ lines, breadth ${ }^{2} / 3$ of a line." Rusticola minor-Darm. Zweifellos gehört diese Art nicht zu Clinostomum.

2) Looss, A., Beitr. zur Kenntn. der Trematoden, in: Z. wiss. Zool., V. 14,1885, p. 427 .

3 Leuckart, R., Die Parasiten d. Menschen, 2. Aufl., V. 1, Abth. 2, p. 40 resp. 154 .

4) Monticelli, F. G., Saggio di una morfologia dei Trematodi, Napoli 1888, p. 92. 
Distomen nicht bekannten Verhältnisse im Genitalapparat der typischen Art (D. reticulatum Looss) bis auf die Lage des Genitalporus völlig zu übergehen. Dadurch wurde Veranlassung gegeben, dass später andere Autoren und Monticelli selbst die verschiedenartigsten Formen der neuen, so bequem zu diagnosticirenden Gattung einreihten, so dass schliesslich Monticeldi ${ }^{1}$ ) eine Liste von 10 Species veröffentlichen konnte, die alle in der Lage des Genitalporus (hinter dem Bauchsaugnapf und vor dem Hinterende) übereinstimmten, aber anatomisch sich erheblich von einander entfernten. Diese Thatsache war Monticellu sehr wohl bekannt, ist ihm auch von BLANCHARD ${ }^{2}$ ) wenigstens für 2 Arten aus dem Menschen (Dist. westermanni Kerb. und Dist. heterophyes v. Sieb.) entgegengehalten worden - aber vergeblich; die Lage des Porus genitalis schien so wichtig, dass Monticeldi in seinem Saggio etc. daraufhin alle Distomen (excl. Rhopalophorus Dies. und Bitharzia Совв.) in 4 Genera theilte (Cephalogonimus PAIR., Distomum Retz., Mesogonimus Mont. und Urogonimus Mont.), später allerdings noch einige andere Genera (Apoblema DuJ., Echinostomum Rud., Crossodera Dus. und Podocotyle Dus., zuliess. Aber das ganze System blieb ein künstliches und sollte auch nur ein solches sein ${ }^{3}$ ).

Schon vorher ergaben die in diese Zeit fallenden Berathungen über die Nomenclaturregeln ein greifbares Resultat; der Wunsch, die in dieser Beziehung gerade innnerhalb der Distomiden bestehende Unsicherheit thunlichst zu beseitigen, liessen Сн. W. Stiles und Alb. Hassall eine kritische Zusammenstellung aller für Distomiden (= Fascioliden) gebrauchten Gattungs- und Untergattungsnamen mit ihren Synonymen publiciren; dadurch, dass für die nach den beiden Autoren giltigen Genusnamen je eine typische Species namhaft gemacht worden ist, ist die Möglichkeit eines weiteren Ausbaues des Systems der Fascioliden gegeben, und hierzu ist von mehreren Seiten durch Aufstellung neuer Genera (durch M. Stossich, Looss, Lühe und mich) beigetragen worden.

Was nun speciell die hier interessirende Gattung anlangt, so stellen

1) Monticelli, F. S., Stud. sui Trematodi endopar. Primo contrib. di osservazioni sui Distomidi, in: Zool. Jahrb., Suppl. 3, Jena 1893, p. 156.

2) Blanchard, R., Note sur quelques vers parasites de l'homme. III. Sur la classific. des Distomiens, in: CR. Soc. biol. Paris, Séance du 18 juill. 1891.

3) Man vergl. Monticelli, F. S., Di un Ematozoo d. Thalassochelys caretta L., in: Internat. Monatsschr. Anat. Physiol., V. 13, 1896. 
Stiles u. Hassall ${ }^{1}$ ) als typische Art Clinostomum gracile Leidy 1856 auf, das sie für identisch mit Distomum heterostomum Rud. 1809 halten; sie glauben sich ferner nach Untersuchung der Originalexemplare LEIDY's überzeugt zu haben, dass mit $\mathrm{Cl}$. gracile wiederum Dist. reticulatum Looss zusammenfällt, und da nun auf letztgenannte Art Mesogonimus Mont. basirt ist, so wird Mesogonimus synonym zu Clinostomum. Wenn nun auch meiner Ueberzeugung nach die Identität der in Rede stehenden Arten bisher nicht erwiesen ist, so handelt es sich in ihnen doch zweifellos um sehr nahe stehende Formen, von denen aber ausreichend nur Dist. reticulatum Looss bekannt ist; die beiden andern Arten ( $C l$. gracile LeIDy und Dist. heterostomum Rud.) sind streng genommen Species inquirendae. Allerdings liegen über sie einige weitere Mittheilungen vor: R. RAMSAY WRIGHT $^{2}$ ) beschreibt eine in der Mundhöhle von Botaurus minor Gr. gefundene Art näher und stellt sie provisorisch zu Distomum heterostomum RuD.; in derselben Arbeit wird noch Clinostomum gracile LEIDY (encystirt bei Perca flavescens Cuv.) beschrieben, aber an einen Zusammenhang dieser Art mit der RudoLPH'schen gar nicht gedacht; der reife Zustand des $\mathrm{Cl}$. gracile, bei dem auch Wright Genitalien nicht gefunden hat (LeIDy erwähnt sie ebenfalls nicht), wird vielmehr im Darm eines Raubfisches oder Fische fressenden Vogels vermuthet; im letztern Fall würde dann zwischen dem encystirten Jugend- und dem reifen Zustand ein solches Verhältniss bestehen, wie es Diesing für Dist. dimorphum annimmt. Man sieht demnach, dass WRIGHT 1879 von der richtigen Auffassung des Cl. gracile als der Jugendform des Dist. heterostomum (resp. einer andern verwandten Art) recht weit entfernt war; doch muss anerkannt werden, dass die Beschreibung dieser Art vieles Richtige enthält.

Eine zweite Notiz über einen Dist. heterostomum RuD. genannten Helminthen erhalten wir durch v. Linstow $\left.{ }^{3}\right)$; die Exemplare stammen

1) Stiles, Ch. W. and A. Hassall, Notes on parasites. 48. An inventory of the genera and subg. of the Tremat. fam. Fasciolidae, in: Arch. Parasitol., V. 1, Paris 1898, p. 81-99.

2) R. Ramsay Wright, Contrib. to Amer. helminthology, in: Proc. Canadian Inst., (new ser.) V. 1, Toronto 1879.

3) v. Linstow, Nematoden, Trematoden und Acanthoceph.. ges. v. Prof.. Fedtschenko in Turkestan, in: Arch. Naturg., Jg. 49, V. 1, 1883, p. 306. - Rund- u. Saugwürmer, Reise nach Turkestan von A. P. FEDTschenko, V. 2, Theil 5, Moskau 1886, in: Verh. Ges. Freunde Naturw., Anthr. u. Ethnogr., V. 34, p. 30, fig. 49. 
aus Ardea nycticorax und werden mit Rücksicht auf WRIGHT's Angaben nur kurz beschrieben. Offenbar sind beide Formen, die canadische und die turkestanische, sehr nahe verwandt, ob jedoch identisch, mag einstweilen dahingestellt bleiben.

Unter dem Namen Distomum reticulatum Looss hat dann auch PoIrIER ${ }^{1}$ ) eine in Axinurus dugesii encystirt gefundene Form kurz beschrieben; auch hier ist die Bestimmung zum mindesten fraglich, doch handelt es sich ebenfalls um eine verwandte Art.

Dies sind die literarischen Angaben, welche meines Wissens Strues u. Hassall bei Abfassung ihres ,Inventory" vorgelegen haben können. So wenig ausreichend sie auch sind, so genügten sie meines Erachtens doch vollkommen, um die Gattung Clinostomum annähernd richtig zu charakterisiren. Das Typische dieser Gattung haben jedoch StILEs u. Hassall nicht herausgefunden, denn in der analytischen Bestimmungstabelle wird als wesentlicher Charakter von Clinostomum $=$ Mesogonimus nur die Lage des Genitalporus (hinter dem Bauchsaugnapf) angegeben und daher neben $\mathrm{Cl}$. gracile auch noch Distomum westermanni KerB. angeführt! Diesen Standpunkt nimmt auch noch W. G. MACCALLUM $^{2}$ ) ein, obgleich er die Anatomie der untersuchten Art (aus der Mundhöhle von Ardea herodias) durchaus richtig darstellt; er unterlässt jedoch den Vergleich mit Dist. westermanni KerB. und stellt daher wie Monticelli, Stiles u. Hassall, diese Art ebenfalls zu Clinostomum.

Nachdem ich mich bereits früher gelegentlich gegen den Gebrauch, alle Distomiden, deren Genitalporus hinter dem Bauchsaugnapf liegt, in eine Gattung zu stellen, ausgesprochen habe, habe ich neuerdings nach genauerem Studium der Clinostomen für diejenigen Arten, welche sich um Dist. westermanni Kerb. gruppiren, den Gattungsnamen Paragonimus vorgeschlagen und Arten mit Dist. leptostomum OLss. als Typus in die Gattung Harmostomum vereint ${ }^{3}$ ). Die Harmostomen sind mit den Clinostomen sehr nahe verwandt, so sehr, dass man

1) Poirier, J., Trémat. nouv. ou peu connus, in: Bull. Soc. philomath. Paris, (sér. 7) V. 10, 1885/86, p. 39.

2 Mac Callum, W. G., On the species Clinostomum heterostomum, in: J. Morph., V. 15, No. 3, 1899, p. 697-710. Die Arbeit ist vom October 1897, das betreffende Heft des Journ. Morph. vom Februar 1899 datirt, es ist jedoch thatsächlich erst im Januar 1900 zur Ausgabe gelangt.

3) Braur, M., Ueber Clinostomum Leidy, in: Zool. Anz., V. 22, 1899 , p. 492. 
beide zu einer Unterfamilie vereinen könnte; Paragonimus aber hat mit dieser Gruppe nichts weiter gemein, nicht einmal die Lage des Genitalporus, der ganz andere Beziehungen z. B. zum Bauchsaugnapf besitzt als bei den Clinostominen. Auch nach dieser Abtrennung bleiben noch Arten übrig, deren Genitalporus ebenfalls hinter dem Bauchsaugnapf liegt; sie sind zum Theil zu wenig bekannt, um sie jetzt schon mit Sicherheit in eine der bestehenden Gattungen einzureihen, resp. um für sie neue Gattungen aufzustellen ${ }^{1}$ ).

Durchaus unabhängig von mir ist A. Looss ${ }^{2}$ ) zu der gleichen Anschauung gelangt; er beschränkt den Begriff Clinostomum auf $\mathrm{Cl}$. gracile resp. dessen Verwandte und creirt für Dist. leptostomum OLss. die Gattung Heterolope, für D. westermanni die Gattung Polysarcus; auch den erwähnten LüHE'schen Aufstellungen entsprechende hat er geschaffen. Diese Uebereinstimmungen dürften für die Natürlichkeit der neuen Gattungen eine gewisse Gewähr bieten, wenngleich damit nicht die Nothwendigkeit einer Begründung wegfällt.

In der Charakteristik der Gattung Clinostomum stimmt nun allerdings Looss nicht ganz mit mir überein, doch erklärt sich dies ungezwungen aus dem Umstande, dass Looss erneute Untersuchungen nicht hat anstellen können, sondern auf seinen vor 15 Jahren an Dist. reticulatum gewonnenen Erfahrungen sowie auf den unzureichenden Beschreibungen anderer Autoren fussen muss, während mir fast das gesammte vorhandene Material an in Betracht kommenden Arten im Jugend- wie im erwachsenen Zustande vorgelegen hat; nur die nordamerikanischen und die turkestaner Formen fehlen mir.

Die Eigenthümlichkeiten der Gattung Clinostomum liegen in der Gestalt des Körpers, in der Beschaffenheit des Vorderendes, im Verhalten des Darmtractus und Excretionssystems, ganz besonders aber im Genitalapparat.

1) Arten, deren Genitalporus neben dem Bauchsaugnapf liegt, hat M. Stossich zum Genus Levinsenia vereinigt und für Distomum turgidum BrDs. die Gattung Brandesia aufgestellt (Lo smembramento dei Brachycoelium, in: Boll. Soc. Adriatica Sc. nat., V. 19, 1899); wogegen LüHe (Zur Kenntniss einiger Distomen, in: Zool. Anz., V. 22, 1899, p. 538) für Dist. lorum Dus. die Gattung Ityogonimus, für $D$. heterophyes die Gattung Cotylogonimus und für D. concavum Crept. die Gattung Cryptocotyle aufgestellt hat.

2) Looss, A., Weitere Beiträge zur Kenntniss der Trematoden-Fauna Aegyptens, zugleich Versuch einer nat. Gliederung des Genus Distomum. Retz., in: Zool. Jahrb., V. 12, Syst., Heft 5/6, 1899, p. 649. 
1) Körpergestalt. Bei allen Arten zerfällt der langgestreckte Körper in zwei verschieden grosse Theile: Vorderleib oder Hals und Hinterleib; die Grenze beider liegt etwa in der Höhe des Bauchsaugnapfes, der stets dem Mundsaugnapf erheblich genähert ist; auch macht sie sich äusserlich durch eine seichte Einkerbung an den Seitenrändern kenntlich. Gewöhnlich ist der Querdurchmesser des Vorderleibes kleiner als der des Hinterleibes, der Dorsoventraldurchmesser dagegen meist grösser. Eine weitere Differenz zwischen beiden Leibesabschnitten liegt darin, dass der gesammte Genitalapparat nur im Hinterleibe liegt, wogegen im Vorderleibe ausser dem Anfangstheil des Darmes und dem Centralnervensystem anscheinend bei allen Arten zahlreiche einzellige Hautdrüsen vorkommen, die am Vorderende ausmünden.

2) Das Vorderende ist ventralwärts schräg abgestutzt, so dass die dorsale Fläche nach vorn etwas weiter reicht als die ventrale; die ganze schräge Fläche nenne ich das Mundfeld; in ihr liegt, wie längst bekannt ist, die Mundöffnung und der von den frühern Autoren meist übersehene, kleine Mundsaugnapf. Das Mundfeld selbst erscheint nun je nach dem Contractionszustand sehr verschieden: es können nämlich die unmittelbar an den Rand anschliessenden Partien eingezogen werden, dann tritt der Rand kragenartig hervor (Fig. 1, $8,11,19)$, und nach innen von ihm zeigt sich eine mehr oder weniger deutliche Ringfurche, die anscheinend auf der Ventralfläche immer etwas tiefer als auf der dorsalen ist. Das Centrum des Mundfeldes habe ich nie ganz eingezogen gefunden, vielmehr hebt es sich in Form eines vorn abgerundeten Zapfens mehr oder weniger empor; auf diesem Zapfen, Mundkegel, liegt dann subterminal der Mund und der Mundsaugnapf. In andern Fällen findet man das Mundfeld ziemlich eben und die Ringfurche verstrichen (Fig. 6, 16) oder aber ersteres ganz vorgewölbt (Fig. 4), doch lässt sich auch in diesem Falle die periphere Begrenzung des Mundfeldes erkennen. Offenbar dient das Mundfeld zum Ansaugen - alle Cliuostomen, die man bis jetzt kennt, bewohnen im erwachsenen Zustande die Mundhöhle oder den Oesophagus und bedürfen besonders kräftiger Saugorgane, um den Schluckbewegungen ihrer Wirthe und dem Andrang der grossen, nicht zerkleinerten Nahrungsbissen zu widerstehen. Während demnach das Vorderende mit dem saugnapfartig wirkenden Mundfeld und dem stets kräftigen Bauchsaugnapf angeheftet ist, kann der Munfsaugnapf die wohl aus Blut bestehende Nahrung ungestört aufnehmen. Spätere Untersuchungen an frischem Material werden zweifellos eine be- 
sondere Anordnung der zum Mundfeld gehenden Musculatur ergeben; ich habe nur gelegentlich zahlreiche, dem Längsmuskelsystem angehörige und sich vielfach theilende Fasern gesehen, die sich an der Peripherie des Mundfeldes inseriren; sie werden bei ihrer Contraction die Ringfurche hervorrufen, während das Hervorwölben des ganzen Mundfeldes wohl ausschliesslich durch die Contraction der Ringmusculatur des Vorderleibes bewirkt wird.

3) Der Darmcanal beginnt mit der Mundöffnung, welche in den Hohlraum des Mundsaugnapfes führt; letzterer setzt sich dann in den Oesophagus fort; in Folge seiner Kürze liegt die Gabelungsstelle des Darmes weit vorn, ziemlich unmittelbar hinter dem Mundsaugnapf resp. dem Mundfeld. Unsicher bin ich über das Vorkommen eines Pharynx; es ist allerdings nicht allzu schwierig, an Totalpräparaten vor der Gabelungsstelle ein ovales Gebilde zu sehen, welches den Eindruck eines Pharynx macht (Fig. 1, 4, 13, 14); mitunter ist aber trotz aller Aufhellungsmittel nichts zu bemerken. Auf einer Schnittserie durch Clinostomum heluans finde ich nichts, was man Pharynx nennen könnte; allerdings erweitert sich der mit dicker Cuticula versehene Oesophagus vor dem Uebergang in den mit hohen und schmalen Cylinderzellen ausgekleideten Darm - aber nach dem Parenchym ist die Erweiterung nicht abgegrenzt, obwohl die nächste Umgebung dieser Stelle etwas anders aussieht; jeden Falls fehlen die sonst so leicht zu sehenden Radiärmuskeln wenigstens bei der genannten Art. Andere Autoren sprechen mit mehr Sicherheit von einem Pharynx bei den Clinostomen, so Ramsay Wright, der allerdings nur sagt: "The pharynx has thin walls, is still smaller"; dagegen zeichnet MaC CALLUm nicht nur einen deutlichen Pharynx bei der von ihm untersuchten Form, sondern sagt auch ausdrücklich im Text, dass dieses Organ ähnlich gebaut sei wie der Mundsaugnapf, der Meridional-, Radial- und Aequatorialfasern erkennen lässt. Wenn dies wirklich beobachtet ist, so würden hier exceptionelle Verhältnisse vorliegen. Ich bin jedoch nach wiederholter Untersuchung von Totalpräparaten und Schnitten zu der Ansicht gekommen, dass ein wirklicher Pharynx den Clinostomen fehlt; was oft genug auf Totalpräparaten als solcher erscheint, ist das erweiterte hintere Ende des Oesophagus, das möglicher Weise eine andere Structur aufweist als der übrige Theil, aber kein Pharynx im gewöhnlichen Sinne des Wortes ist.

Die Darmschenkel ziehen gewöhnlich zuerst ein kurzes Stück in querer Richtung, ja nicht selten sogar auch etwas nach vorn, biegen 
dann recht- resp. spitzwinklig nach hinten um und verlaufen ungefähr parallel den Seitenrändern des Körpers bis ans Ende des Hinterleibes. Bei einer Art, die ich für das typische Distomum heterostomum RuD. halte, ziehen sie verhältnissmässig nahe der Mittellinie nach hinten (Fig. 2). Wohl bei allen Arten sind die Darmschenkel wenigstens im Hinterleibe mit kurzen, mehr oder weniger dicht stehenden, nach innen wie nach aussen gerichteten Blindsäckchen versehen, die den Contouren ein halskrausenartiges Aussehen verleihen (Fig. 4, 5, 7, 10, 16, 17). Seltener beginnt diese Haustrirung der Darmschenkel schon im Vorderende (Fig. 16, 17), und nur bei einer Art, derselben, bei der sie der Mittellinie genähert verlaufen, finden sich jederseits 9-11 lange, nach aussen und hinten gerichtete, manchmal am Ende gegabelte Blindschläuche (Fig. 2).

4) Das Excretionssystem lässt sich bei den erwachsenen Exemplaren, wenn überhaupt, nur in einzelnen Abschnitten verfolgen; leichter ist dies bei den kleinern und durchsichtigern Jugendstadien, bei denen manchmal grosse Strecken besonders des oberflächlich gelegenen Netzwerks an ihrem geronnenen, fein granulirtem Inhalt deutlich hervortreten (Fig. 19). Diese an das Verhalten von Fasciola hepatica L. erinnernden Verbindungen eines bestimmten Abschnittes der Excretionsorgane hat bereits Looss erkannt und deshalb der von ihm untersuchten Art den präoccupirten Namen, ,reticulatum" gegeben. Ausser diesem oberflächlich gelegenen Netzwerk, dessen Maschen polygonal oder quer gestreckt sind, bemerkt man zu den Seiten des Körpers, aber tiefer im Innern, je eine oder zwei Sammelröhren, die das Secret wohl direct in die in ihrer Form mir nie deutlich genug gewordenen Excretionsblase führen werden. MACCALLUm unterscheidet ausser den Terminalzellen und Capillaren noch das subcuticulare Netzwerk, die wie dieses bewimperten Seiten- oder Sammelröhren und eine paarige Endblase, die durch einen Porus ausmündet.

5) Der Genit alap parat ist für die Clinostomen ganz besonders charakteristisch; seine wesentlichsten Eigenthümlichkeiten hat bereits Looss bei Dist. reticulatum erkannt. Dass der gesammte Genitalapparat im Hinterleibe, also hinter dem Bauchsaugnapf liegt, ist bereits angeführt. Der meist flachere und breitere Hinterleib lässt gewöhnlich 3 etwa gleich breite Längsfelder erkennen; die beiden seitlichen sind von den Dotterstöcken (und Darmschenkeln), das mittlere von den übrigen Geschlechtsorganen eingenommen.

In oder hinter der Mitte des Mittelfeldes, jedoch meist nicht ganz im Hinterende, finden wir die beiden Hoden, den Keimstock und 
die Schalendrüse in ganz bestimmten und regelmässig wiederkehrenden Lagebeziehungen zu einander; die von diesen Organen eingenommene Partie des Mittelfeldes will ich Genitaldrüsenfeld nennen. Die Hoden sind nie ganzrandig, sondern mehr oder weniger eingekerbt oder tief eingeschnitten, so dass sie lappig oder strahlig erscheinen. Gewöhnlich ist ihr Querdurchmesser erheblich grösser als der Längs- und Dorsoventraldurchmesser; diese beiden Organe liegen hinter einander und begrenzen mit ihrem Vorder- resp. Hinterrande das Genitaldrüsenfeld vorn und hinten; die einander zugekehrten Hinter- resp. Vorderflächen der beiden Hoden sind in der Regel ziemlich eben oder weniger tief eingeschnitten als die von einander weg gewendeten und begrenzen einen meist rechteckigen, quer gestellten Raum, in welchem rechts der kleine, rundliche Keimstock liegt; neben diesem, aber den grössten Theil des Raumes zwischen den Hoden einnehmend, finden wir den sich stark windenden Anfangstheil des Uterus, umgeben von grossen Schalendrüsenzellen, und dahinter in der Mitte des Vorderrandes des hintern Hodens das Dotterreservoir, zu dem von den Seiten die queren Dottergänge hinstreben. Ausnahmsweise liegt auch noch in dem von den Hoden begrenzten Raum der Genitalporus und der Cirrusbeutel (Fig. 13, 14); die gewöhnliche Lage für den letztern ist die rechte Körperseite am rechten Vorderrande des vordern Hodens, also vor dem ebenfalls rechts gelegenen Keimstock (Fig. 4, 6, 10, 11), seltner die Mittellinie vor dem vordern Hoden (Fig. 1, 2). Dem entsprechend variirt auch die Lage des Genitalporus je nach den Arten: er liegt gewöhnlich unmittelbar vor dem vordern Hoden, entweder etwas rechts von der Mittellinie oder in dieser selbst, selten zwischen den Hoden.

Noch grössere Eigenthümlichkeiten bietet der Uterus; es ist schon bemerkenswerth, dass sein zwischen den Hoden gelegener Anfangstheil innerhalb des Complexes der Schalendrüse so zahlreiche Windungen macht, noch sonderbarer aber ist es, dass die Fortsetzung dieses Ganges, dessen Lichtung nicht weiter ist als der Querdurchmesser der Eier, sich links hin wendend nach vorn vor den vordern Hoden gelangt und hier von der Seite in einen bedeutend weitern, sich bis zum Bauchsaugnapf erstreckenden und dort blind endigenden, lang gestreckten Raum einmündet (Fig. 4, 5), in dem die grossen Eier sich anhäufen (Fig. 20). Dieser von mir Uterussack genannte Raum steht an seinem hintern Ende mit dem Genitalporus in Verbindung.

Mit Recht hat Looss, der auch diese höchst bemerkenswerthen 
Verhältnisse richtig erkannt hat, den Uterussack mit dem allerdings keine äussere Mündung besitzenden Uterus einer Cystotänie verglichen; in beiden Organen häufen sich die Eier an, und zu beiden zieht von der weiblichen Geschlechtsdrüse her ein mehr oder weniger langer Gang, den ich bei den Tänien Uteringang genannt habe. Die Aehnlichkeit des Uterussackes der Clinostomen mit dem Uterus der Tänien erhöht sich noch dadurch, dass ersterer bei einigen Arten im Laufe der Zeit kurze, seitliche Ausbuchtungen, event. selbst Sprossen treibt, die ihm ein durchaus tänioides Aussehen verleihen (Fig. 11).

Sonst ist bekanntlich der Uterus der Distomen ein im Grossen und Ganzen gleich weites Rohr, das zwar sehr verschieden verlaufen kann, aber doch wohl niemals in zwei so differente Abschnitte wie bei den Clinostomen zerfällt; freilich ist oft der Endtheil des Uterus, das Metraterm, von einigen Autoren auch wohl Vagina genannt, durch besondere Structurverhältnisse der Wandung ausgezeichnet, aber dieser Theil behält doch die ursprüngliche Weite des ganzen Canales bei und setzt sich daher nicht so scharf ab.

Bei der weit nach hinten gerückten Lage des Genitalporus sollte man erwarten, dass der Uterus einem umgekehrten $\mathrm{U}$ gleicht, d. h. nach seinem Ursprung in einem aufsteigenden Schenkel nach vorn, etwa bis zum Bauchsaugnapf zieht und hier dann bogenförmig in den absteigenden, durch den Porus ausmündenden Schenkel übergeht. Ein solcher Uterus kommt den Arten der Gattung Harmostomum (Typus: Dist. leptostomum OLss.) $\mathrm{zu}^{1}$ ) und stellt jeden Falls das ursprüngliche Verhalten dar. Schon in meiner vorläufigen Mittheilung habe ich darauf hingewiesen, dass ich etwas Aehnliches auch bei einer Clinostomum-Art, dem $\mathrm{Cl}$.heterostomum (RUD.), beobachtet habe, hier steigt nämlich der dünne Uterustheil als gerader Gang auf der linken Körperseite hinauf bis an den Hinterrand des Bauchsaugnapfes, biegt hier nach der Mittellinie zu im Bogen um und geht nun in den weit breitern Uterussack über (Fig. 1, 2). Durch Herabrücken dieser Einmündungsstelle lässt sich das gewöhnliche Verhalten bei Clinostomen erklären.

Dass auch ein LAURER'scher Canal vorkommt, berichtet schon Looss, MacCallum erwähnt ihn ebenfalls, und ich habe ihn wenigstens bei $\mathrm{Cl}$. heluans gefunden.

1) Vergl. Hofmann, K., Beitr. zur Kenntn. der Entwicklung von Dist. leptostomum OLss., in: Zool. Jahrb., V. 12, Syst., 1899, p. 174 -204 . 
Noch ist anzuführen, dass der Raum des Mittelfeldes hinter dem hintern Hoden ebenfalls von Dotterstocksfollikeln eingenommen wird, wie denn überhaupt die Dotterstöcke bei den Clinostomen sehr stark entwickelt sind; sie dehnen sich medianwärts bis an den Uterussack und das Genitaldrüsenfeld und lateral bis fast an den Seitenrand aus; nur eine ganz schmale Seitenzone bleibt frei. Die Dotterstocksfollikel sind gewöhnlich recht gross, und eine besondere Anordnung in Gruppen macht sich nicht bemerklich; bei $C l$. dimorphum s. str. sind die Follikel ziemlich klein und befinden sich in reticulärer Anordnung.

Nach diesen Mittheilungen ist es wohl überflüssig, näher auseinander zu setzen, dass die Clinostomen mit keiner derjenigen Arten resp. Artengruppen, welche den Genitalporus neben oder hinter dem Bauchsaugnapf tragen, in näheren Beziehungen stehen; ein Blick auf die in der Literatur vorhandenen Beschreibungen und Abbildungen der Typen solcher Artengruppen lässt ohne weitere Ausführungen die grossen Differenzen sofort erkennen. Nur eine Artengruppe macht hiervon, wie bereits erwähnt, eine Ausnahme, das sind die von mir zu Harmostomum, von Looss zu Heterolope vereinten Arten: D. leptostomum Olss., D. spinosulum Hofm., D. opisthotrias Lutz und $H$. aequans Looss (aus Gerbillus aegyptius). Bei allen Harmostomen liegt der Genitalporus in der Mittellinie der Bauchfläche weit hinter dem Bauchsaugnapf am Vorderrand des vordern Hodens; auch hier ist das von den Darmschenkeln begrenzte Mittelfeld in dem vordern größern Abschnitt vom Uterus, in dem kleinern hintern von dem Hoden und dem Keimstock eingenommen. Diese drei Drüsen folgen hinter einander derart, dass zwischen den Hoden der Keimstock liegt; der Uterus verläuft zunächst nach vorn bis vor den Bauchsaugnapf und biegt hier in den absteigenden Schenkel um. Jedoch unterscheiden sich die Harmostomen schon durch die Körpergestalt von den Clinostomen; ihr Körper ist fast walzenförmig, sehr langgestreckt, von ovalem oder fast kreisrundem Querschnitt; niemals sondert sich der Vorderleib von dem Hinterleibe, und niemals begegnet man unter den Harmostomen einer Ausbildung des Vorderendes, wie sie die Clinostomen regelmässig besitzen; Mund- und Bauchsaugnapf sind fast gleich gross, und der Eingang in den erstern stellt einen längs gestellten Spalt dar. Des weitern besitzen die Harmostomen einen typisch entwickelten Pharynx, wogegen die ebenfalls bis ins äusserste Hinterende reichenden Darmschenkel nie mit Blindsäckchen besetzt sind. Auch das Gefässystem bietet Differenzen, vor allem 
aber auch trotz aller Aehnlichkeit der Genitalapparat: die Hoden sind rundlich, eher in die Länge als in die Quere entwickelt, der Keimstock liegt in der Mittellinie, nicht rechts, die Schalendrüse ist compacter und rechts vor dem Keimstock gelegen; weit schwächer sind die Dotterstöcke entwickelt, und der Uterus bildet nie einen sackartig erweiterten Endabschnitt, sondern ist überall wie gewöhnlich gleich weit; auch ist die Zahl der Eier bei den Harmostomen weit grösser, die Eier selbst sind erheblich kleiner und von andrer Gestalt; endlich leben die Harmostom en im Dünndarm von Säugern (Erinaceus, Didelphys und Gerbillus), die Clinostomen im Vorderdarm fischfressender Vögel (Ciconier).

Looss vereinigt mit Heterolope $=$ Harmostomum die neue, auf Distomum lorum DuJ. (aus Talpa europaea) basirte Gattung Dolichosomum (=Ityogonimus LüHE) zu einer Unterfamilie: Heter olopinae, und will hierdurch die nach ihm bestehende nahe Verwandtschaft beider Gattungen ausdrücken. Distomum lorum DuJ. ist aber trotz der Arbeit von MeLniKow ${ }^{1}$ ) doch wohl noch nicht so genau bekannt, dass man dies jetzt schon als ganz sicher annehmen könnte; immerhin ist es wahrscheinlich; jeden Falls wird aber die Unterfamilie nicht den Namen Heterolopinae tragen können, da Harmostomum Priorität vor Heterolope besitzt und doch wohl auch trotz aller Differenzen auch die Clinostomen anzuschliessen sind.

\section{Arten der Gattung Clinostomum.}

Bevor ich die Arten beschreibe, dürfte es zweckmässig sein, einen Ueberblick über die bisher bekannten Arten zu geben; dabei scheide ich von vorn herein alle Arten, die sicher nicht Clinostomen sind, aus, ebenso einstweilen auch die nur im Jugendzustande bekannten, die im Zusammenhang mit dem mir vorliegenden Material weiter unten besprochen werden sollen.

Als Typus für die Gattung Clinostomum wird übereinstimmend von Stiles u. Hassall sowie von Looss Distomum heterostomum RuD. angenommen; unglücklicher Weise ist nun diese Art ebenso wenig genau bekannt wie ihr vermeintlicher Jugendzustand: $C l$. gracile Leidy, mit dem trotz der Ansicht von Stiles u. Hassall Distomum reticulatum Looss nicht identisch ist.

1) Melnikow, N., Ueber das Dist. lorum Duj., in: Arch. Naturg., Jg. 31, V. 1, 1865, p. 49-55. 
Wir finden Dist. heterostomum Rud. zuerst bei Rudolphi ${ }^{1}$ ) beschrieben; 2 Exemplare hatte A. Jurine im Oesophagus von Ardea purpurea gefunden und eins an RudolpHi gesandt. Der betreffende Wurm war $6,7 \mathrm{~mm}$ lang, in der Mitte des Körpers über 2,25 mm breit und von weisser Farbe; Bauchfläche eben, Rückenfläche etwas gewölbt, die Seitenränder abgestumpft; etwa der vierte Theil der Gesammtlänge fiel auf den Hals, welcher etwas schmäler als der übrige Körper war. Vorn bemerkte man auf der Unterfläche einen grossen Porus mit dreieckiger Mündung und etwas verdicktem Rand; der Bauchsaugnapf war dem Porus sehr genähert, kleiner und tiefer als dieser und besass einen länglichen, beinahe dreieckigen Eingang. In der Verdindungslinie beider Pori, aber 2,25 mm hinter dem Bauchsaugnapf, also etwa in der Mitte der ganzen Körperlänge, fand sich eine dritte, sehr kleine, kreisförmige Oeffnung, die ohne Zweifel zum Austritt des im Innern verborgen liegenden Cirrus dient. Im Uebrigen war der Körper des in Alkohol conservirten Exemplars mit dicht stehenden, parallelen Querstreifen versehen, innere Organe aber nicht sichtbar.

In der "Synopsis" (p. 102 und 388) giebt RudolPHI eine nur unwesentlich veränderte Diagnose dieser Art und zieht $\mathrm{zu}$ ihr auch diejenigen Würmer, welche RosA ${ }^{2}$ ) in der Mundhöhle von Ardea purpurea gefunden und als Leberegel angesehen hatte; die Thiere waren $6,75 \mathrm{~mm}$ lang, in der Mitte 2,25 $\mathrm{mm}$ breit, weiss, quer gestreift und wiesen jederseits eine dunkle Längslinie auf.

DUJARDIN ${ }^{3}$ ) wiederholt nur die Beschreibung bei RudolPHI, bezweifelt jedoch die Richtigkeit der Angabe über die Lage des Genitalporus und thält Dist. heterostomum Rud. überhaupt für eine zweifelhafte Species, die ebenso wie Dist. complanatum Rud. zu Dist. hians RuD. gehöre. Diesing ${ }^{4}$ ) dagegen führt alle drei Arten gesondert auf, ohne etwas Neues hinzuzufügen.

Provisorisch zu Dist. heterostomum Rud. stellt Ramsay W $\mathrm{RIGHT}^{5}$ ) in der Mundhöhle von Botaurus minor Gs. gefundene Trematoden; er erkannte, dass die von RudoLPHI als Porus anticus angesehene

1) Rudolphi, C. A., Entozoorum Hist. nat., V. 2, Ps. 1, Amstelod. 1809, p. 381.

2 Rosa, V., Lett. zool., Pavia 1794, No. 5, p. 5.

3 Dujardin, F., Hist. nat. des helminthes, Paris 1845, p. 400.

4) Diesing, C. M., Syst. helm., V. 1, Vindobonae 1850, p. 353.

5) Ramsay Wright, Contr. Amer. helm., in: Proc. Canad. Inst., (new ser.) V. 1, 1879, p. 3. 
vertiefte Stelle am Vorderende nicht der Mundsaugnapf sei, sondern dass dieser auf einem aus der Vertiefung hervorragenden Zapfen liege; er ist erheblich kleiner als der Bauchsaugnapf ( 0,3 gegen $0,8 \mathrm{~mm})$; die ganze Körperlänge beträgt 6,85 , die grösste Breite $1,5 \mathrm{~mm}$, doch können die Thiere auch eine mehr gestreckte Form annehmen; der Pharynx ist dünnwandig und klein, hinter ihm treten sofort die beiden, wegen ihres braunen Pigmentes leicht erkennbaren Darmschenkel ab, welche hinter dem Bauchsaugnapf bis zum Hinterende mit kleinen Divertikeln besetzt sind. Die Analyse der Geschlechtsorgane ist WrIGHT jedoch nicht in allen Punkten gelungen; richtig wurden die Dotterstöcke und die Hoden erkannt; die Eier sind 0,099 mm lang, $0,066 \mathrm{~mm}$ breit.

Ebenfalls unzureichend ist die Beschreibung einer als Dist. heterostomum Rud. bezeichneten Art durch V. Linstow ${ }^{1}$ ); Fedtschenko hatte sie in Ardea nycticorax $=$ Nycticorax griseus in Turkestan gesammelt; die Thiere waren $3 \mathrm{~mm}$ lang, $1,5 \mathrm{~mm}$ breit; als Grösse des Mundsaugnapfs, wofür aber das ganze Mundfeld angesehen wurde, wird 0,66, als die des Bauchsaugnapfs $0,62 \mathrm{~mm}$ angegeben. Theile des Gefässnetzes, die Hoden, der Keimstock, Dotterstöcke und Uterussack wurden erkannt; die Grösse der Eier betrug 0,11:0,079 mm.

Erwähnt wird dann von C. PARONa ${ }^{2}$ ) Dist. heterostomum Rud. aus dem Oesophagus von Nycticorax griseus (Sardinien) - jedoch unter Dist. hians Rud., wozu auch $D$. complanatum Rud. nach dem Vorgange Dujardin's gezogen wird; ferner von Stossich ${ }^{3}$ ) aus dem Oesophagus von Nycticorax griseus und von C. PARona ${ }^{4}$ ) aus Ardea purpurea. Endlich ist dann noch die Arbeit MaCCALLun's ${ }^{5}$ ) hinzugekommen, dessen Exemplare, wie die Wright'schen, aus Canada, jedoch aus einem andern Wirth (Ardea herodias) stammten. Sie waren 6-10 $\mathrm{mm}$ lang, 1-2 $\mathrm{mm}$ breit, roth gefärbt, die Darmschenkel schimmerten als braune Streifen durch, wogegen Hoden und Uterus als weissliche Massen erschienen. Diese Form stimmt mit der von

1) v. Linstow, in: Arch. Naturg., Jg. 49, V. 1, 1883. p. 306. Reise nach Turkestan, Moskau 1886.

2) Parona, C., Elmintol. Sarda, in: Ann. Mus. civ. Genova, (ser. 2) V. 4, 1887, p. 331.

3) Stossich, M., Observ. elmint., in: Soc. Hist. nat. croat., Ann. 7, 1892 , p. 64.

4) Parona, C., Elm. del Mus. zool. di Torino, in: Boll. Mus. Zool. Anat. comp. Torino, V. 11, No. 258, 1896.

5) MacCallum, W. G., in: J. Morph., V. 15, 1899, p. 697. 
R. Wright untersuchten Art überein; ihre Eier sind 0,099 mm lang, $0,066 \mathrm{~mm}$ breit. Beiläufig erfahren wir noch durch STILES u. HasSALL $^{1}$ ) bei Gelegenheit der Beschreibung von Dist. complexum ST. et H., dass auch Dist. aquilae LEIDY mit Dist. heterostomum RuD. identisch sein soll; diese Art fand LeIDY ${ }^{2}$ ) in 2 Exemplaren in der Trachea von Haliaëtus lencocephalus und beschreibt sie in drei Zeilen; es ist bezeichnend, dass der Autor die Zugehörigkeit dieser Form zu Clinostomum nicht erkannt hat, sie ist aus der Beschreibung nicht einmal zu vermuthen.

Schon aus diesen Angaben dürfte hervorgehen, dass unter Dist. heterostomum RuD. mehrere Arten in der Literatur gehen; es wird daher zu prüfen sein, ob die Beschreibungen, eventuell wo solche fehlen, $o b$ die Originalexemplare mit der Beschreibung bei RudoLPHI übereinstimmen. Dass die Wright'schen und MaCCallum'schen Exemplare unter einander übereinstimmen, unterliegt keinem Zweifel; diese Form erscheint mir aber zu schlank, als dass von ihr RudolpHI die nun einmal vorhandene Beschreibung hätte liefern können; die Länge wird von Wright mit 6,85, von MacCallum mit 6-10, die Breite mit 1,5 resp. $1-2 \mathrm{~mm}$ angegeben; offenbar sind nun gestreckte Thiere schmäler als zusammengezogene, so dass die $6 \mathrm{~mm}$ langen ca. $2 \mathrm{~mm}$, die $10 \mathrm{~mm}$ langen nur $1 \mathrm{~mm}$ breit sind; bei dem RudoLPHI'schen Exemplar dagegen betrug die grösste Breite über 1 Linie, d. h. über $2,25 \mathrm{~mm}$ bei einer Länge von ca. $6,75 \mathrm{~mm}$ (=3 Linien). Ferner ist der Halstheil bei den canadischen Exemplaren kaum schmäler als der Hinterleib, bei dem RudoLPHI'schen wenig schmäler, aber deutlich ausgebildet, der Scheitel ist hier abgerundet, dort abgestutzt; RUDOLPHI spricht weiterhin von dicht stehenden parallelen Querstreifen, die, wenn sie zu jener Zeit erkannt werden sollten, nicht allzu fein sein konnten; das setzt aber wiederum eine gewisse Dicke und Derbheit des Körpers voraus, welche die canadischen Exemplare nicht besitzen, die aber RUdoLPHI ausdrücklich angiebt: ,substantia vermis solidior et crassior"; daher ist auch nichts von innern Organen gesehen worden. Auch die Form des Einganges des Bauchsaugnapfs ist eine andere: rundlich oder schildförmig bei den canadischen, längsgestreckt bei dem Rudolphi'schen Exemplar; endlich meine ich auch,

1) Stiles, Ch. W., and A. Hassall, Notes on parasit. 21, in: Veterin. Mag., June 189,4 p. 414 Anm.

2) Leidy, J., Notice on some paras. worms, in: Proc. Acad. nat. Sc. Philadelphia, 1887, p. 24. 
dass Rudolphi den Genitalporus nur bei einer massigeren Form hat sehen können.

Gern gebe ich zu, dass dies alles nur Wahrscheinlichkeitsgründe, aber keine Beweise sind; solche wären nur durch Nachuntersuchung des Originalexemplars zu erbringen, doch ist dieses in der Berliner Sammlung nicht mehr vorhanden; unbekannt ist mir, ob die von JuRIne angefertigte Zeichnung, die RudoLPHI (l. c.) erwähnt, noch existirt. Jeden Falls dürfte aber ein Umstand dieselbe Beweiskraft besitzen wie die Nachuntersuchung des Originals, das ist die Thatsache, dass eine mit der canadischen genau übereinstimmende Form bisher in Europa nicht aufgefunden ist, wohl aber eine, und zwar wiederholt, auf welche die RudoLPHI'sche Beschreibung des Dist. heterostomum vollständig passt. Sie liegt mir aus Ardea purpurea von Genua und Turin, aus Ardea cinerea von Pavia und aus Nycticorax griseus von Cagliari und von Zaule bei Triest vor. Damit schweben aber auch alle Synonyme zu Dist. heterostomum Rud., welche von Stiles u. Hassall sowie von MacCallum angegeben sind, einstweilen in der Luft.

2) Eine andere hierher gehörige Art ist Dist. complanatum Rud. Die betreffenden Exemplare wurden von Rosenthal zu Berlin im Oesophagus eines Reihers (Ardea cinerea) gesammelt und von RuDOLPHI ${ }^{1}$ ) beschrieben; die Art ist kleiner, 3,4-5,6 mm lang, kaum $1,13 \mathrm{~mm}$ breit, weiss mit schwarzen Punkten, aber doch so durchsichtig, dass die Dotterstöcke mit einem sie durchziehenden braunen Gefäß (Darmschenkel) erkannt werden konnten. Die Saugnäpfe liegen sehr nahe an einander, die Mündung des hintern ist bald länglich, bald dreieckig. Der Körper selbst ist länglich, dünn, vorn etwas verjüngt, hinten abgestutzt. Die Aehnlichkeit mit Dist. heterostomum entgeht RuDOLPH nicht, doch ist es von diesem durch andere Körpergestalt und das Verhalten der Saugnäpfe unterschieden; allerdings weist RudoLPHI auch auf Dist. hians hin, wohl wegen des gleichen Wohnsitzes in nahe verwandten Wirthen. Dieser Hinweis mag DuJardin (l. c.) bewogen haben, alle drei Arten für identisch zu halten. In der Literatur wird das Auffinden von Dist. complanatum nur noch einmal erwähnt und zwar durch C. PARona ${ }^{2}$ ), der einige Exemplare in Ardea cinerea gefunden hat; eine Beschreibung wird nicht gegeben. Da die

1) Rudolphi, C. A., Entoz. synops., Berol. 1819, p. 98 u. 376.

2) Parona, C., Vermi parasit. in anim. della Liguria, in: Ann. Mus. civ. Genova (ser. 2) V. 4, 1887, p. 489. 
Originalexemplare beider Autoren vorhanden sind, kann die Art genauer charakterisirt werden.

3) Durch v. Olfers erhielt RudolphI Trematoden, welche in Brasilien im Rachen eines als Ardea No. VI bezeichneten Vogels gesammelt waren, wo sie schaarenweise am Gaumen und an den Seiten der Zunge sassen. Diese Art wird von RudoLPHI ${ }^{1}$ ) unter dem Namen Dist. marginatum beschrieben und als ihre nächsten Verwandten $D$. heterostomum und $D$. complanatum (neben $D$. hians) bezeichnet. Sie erreicht 6,75-9 $\mathrm{mm}$ und darüber an Länge; der Hals ist 1,68, der Körper 3,14 mm breit; Bauchfläche eben, Rückenfläche gewölbt, vorn wie hinten abgestutzt. Mundfeld bauchständig, fast nierenförmig mit kleiner kreisrunder Eingangsöffnung; Bauchsaugnapf etwas entfernt, dick, Mündung dreieckig; hinter ihm in der Mitte des Körpers eine eingesunkene Stelle, wo der Cirrus verborgen liegt, der in einem getrockneten Exemplar hier durchschimmerte. Die Darmschenkel und Dotterstöcke sind auch hier gesehen worden.

Unter den Namen Dist. marginatum geht in der Literatur noch eine zweite, von MoLIN ${ }^{2}$ ) aufgestellte Art aus Anas crecca, welche jedoch mit der RudoLPHI'schen Species nichts zu thun hat und umbenannt werden muss.

4) Distomum dimorphum Dies. Unter den von NATterer in Brasilien gesammelten Helminthen befanden sich auch zahlreiche theils aus Fischen, theils aus dem Rachen resp. Oesophagus mehrerer Ardea- und Ciconia-Arten stammende Trematoden, welche alle Diesing zusammen mit Dist. marginatum Rud. für ein und dieselbe Art hielt. Sie erhält den Namen Dist. dimorphum ${ }^{3}$ ); von dieser Art werden zwei Formen unterschieden: die eine kleinere (forma primaria) kommt encystirt in Fischen und im Rachen resp. Oesophagus von Vögeln vor, die andere (forma secundaria) erreicht in Ardea cocoi eine Länge von ca. $100 \mathrm{~mm}$. Dieselben Formen hat Dtesing in einer spätern Arbeit auch abgebildet ${ }^{4}$ ). Dass hierunter mehrere Arten enthalten sind, habe ich in meinen vorläufigen Mittheilungen bereits erwähnt. Nicht identisch mit Dist. dimorphum Dies. ist Dist.dimorphum

1) Rudolphi, C. A., Entoz. synops., Berol. 1819, p. 680.

2) Molin, R., Prodrom. faun. helm. Venetae, in: Denkschr. Akad.

Wiss. Wien, math.-nat. Cl., V. 19, 1861, p. 189-338.

3) Diesing, C. M., Syst. helm., V. 1, Vindob. 1850 , p. 353.

4) Derselbe, 19 Arten von Trematoden, in: Denkschr. Akad. Wiss. Wien, math.-nat. Cl., V. 10, 1855. 
WAgEner $^{1}$ ) aus dem Coecum des Haushuhns, Diesing ${ }^{2}$ ) nennt diese Art Dist. commutatum, und Sonsino ${ }^{3}$ ) beschreibt sie etwas genauer; sie scheint mit den Harmostomen verwandt zu sein.

\section{Beschreibung der Arten von Clinostomum LEID.}

1. Clinostomum heterostomum (RuD.).

(Taf. 1, Fig. 1 u. 2.)

? 1794. Fasciola epatica Rosa, Lett. zool., p. 5, No. 5.

1809. Distoma heterostomum RudolpHi, Ent. Hist. nat., V. 2, p. 381.

1819. Distoma heterostomum Rudolphi, Ent. Synops., p. 102 u. 388.

1845. Distoma heterostomum DuJardin, Hist. nat. helm., p. 400.

1850. Distomum heterostomum Diesing, Syst. helm., V. 1, p. 353.

1887. Distoma hians p. p. Parona, in: Ann. Mus. civ. Genova (ser. 2) V. 4, p. 331 (aus Nycticorax griseus).

1892. Distomum heterostomum Sтоssich, in: Soc. hist.-nat. Croat., Ann. 7, p. 64.

1896. Dist. (Dicrocoelium) heterostomum Parona, in: Boll. Mus. Zool. Anat. comp. Torino, V. 11, No. 258.

1899. Clinostomum heterostomum Braun, in: Zool. Anz., V. 22, p. 489.

1900. Clinostomum heterostomum Braun, in: Ctrbl. Bakt., V. 27, Abth. 1, p. 30.

Wie bereits erwähnt, ist das einzige Exemplar, welches RudouphI vorlag, nicht mehr vorhanden; meine Beschreibung stützt sich auf Exemplare folgender Herkunft:

1) 1 Exemplar aus Ardea cinerea; Genueser Sammlung; bezeichnet als Dist. complanatum (= D. hians), Pavia 23./3. 1880.

2) 1 Exemplar aus Nycticorax griseus; Genueser Sammlung; bezeichnet als Dist. complanatum (= D. hians), Cagliari, 29./4. 1881.

3) 3 Exemplare aus Ardea purpurea; Genueser Sammlung; bezeichnet als Dist. heterostomum, Genua, 13./5. 1887.

4) 1 Exemplar aus Nycticorax griseus; Triestiner Sammlung; bezeichnet als Dist. heterostomum? Zaule, 24./4. 1891.

5) 2 Exemplare aus Ardea purpurea; Turiner Sammlung; bezeichnet als Dicrocoetium heterostomum R. (Inv. No. 28, C. No. 359),

1) Wagener, G., Enthelminthica III, in: Arch. Anat. Physiol, Jg. 1852 , p. $555-569$.

2) Diesing, C. M., Rev. d. Myzhelm. Abth. Tremat., in: SB. Akad. Wiss. Wien, math.-nat. Cl., V. 32, 1858, p. 307-390.

3) Sonsino, P., Studi e notizie elmintol., in: Proc. Verb. Soc. Tosc. Sc. nat., 7 luglio 1889. 
ausserdem mit der handschriftlichen Bezeichnung von DE FILIPPI: „Distoma affin. all' heterostomum dell' esofago dall' Ardea purpurea." (In demselben Glas befanden sich noch 2 Exemplare, von denen eins zu Clinost. foliiforme gehört.) Jeden Falls sind dies jetzt die ältesten Exemplare von Dist. heterostomum.

Alle Exemplare, deren Uebersendung ich den Herren Camerano in Turin, Parona in Genua und Stossich in Triest verdanke, stimmen völlig unter einander überein und sind, wenn man Uebung im Unterscheiden der Clinostomen erlangt hat, bereits mit unbewaffnetem Auge sicher zu erkennen. Die Thiere sind (in Alkohol) weisslich, derb und hart anzufühlen; ihre Bauchfläche ist eben, die Rückenfläche sanft gewölbt; Vorderleib (Hals) und Hinterleib sind durch eine deutliche Einziehung an den Seitenrändern, welche in oder hinter der Mitte des dickwulstigen Bauchsaugnapfs liegt, von einander gesondert. Der Hals selbst ist etwas schmäler als der zungenförmige Hinterleib, in der Vitte ein wenig breiter als vorn und hinten und die Ränder stumpfer.

Die Körperlänge schwankt zwischen 6,7 und $9 \mathrm{~mm}$, die Breite beträgt in der Mitte des Halses etwa $2,5 \mathrm{~mm}$ und in der Höhe des Genitaldrüsenfeldes ungefähr $3,5 \mathrm{~mm}$. Je nach der Contraction nimmt der Hals ein Drittel bis ein Viertel der gesammten Körperlänge in Anspruch. Mit unbewaffnetem Auge sind fernerhin noch das längs oder quer ovale, mitunter mehr dreieckige Mundfeld, der dickwulstige Bauchsaugnapf und die Querstreifen zu sehen, die RudoLPHI erwähnt; zur Erkennung des Genitalporus, des Mundkegels und der Mundöffnung bedarf es meist einer stärkern Lupe; aber auch mit Hülfe dieser ist von innern Organen nichts zu bemerken.

Die weitere Untersuchung geschah mit dem Mikroskop nach Aufhellung in Glycerin oder Creosot; namentlich bei Anwendung des letztern Mittels ist es nothwendig, da es schliesslich sehr stark wirkt, die Untersuchung schon bald nach dem Ueberführen in Creosot zu beginnen, event. muss man stark abblenden und schräge Beleuchtung anwenden; von der Anfertigung von Schnittserien habe ich nicht nur wegen der Seltenheit des Materials, sondern auch deshalb abgesehen, weil nach meinen Erfahrungen ältere Objecte sich kaum distinct färben lassen und die ganzen aufgehellten Thiere die innern Organe ohne Schwierigkeiten erkennen lassen; allerdings bleibt hierbei manches Detail verborgen, das müssen Untersuchungen an frischem Material nachtragen.

Wie schon Ramsay Wright (l. c.) betont hat, haben die Autoren 
vor ihm (zum Theil auch nach ihm) das Mundfeld für den Mundsaugnapf gehalten; bei eingezogenem Mundkegel ist das um so eher möglich, als die wulstigen Ränder des Mundfeldes dann eine Vertiefung begrenzen, in welcher die wirkliche Mundöffnung nur schwer zu sehen ist. Bei Clin. heterostomum ist dieselbe noch dazu klein $(0,233 \mathrm{~mm}$ im Querdurchmesser) und auffallend weit ventralwärts verschoben; sie liegt wie überall auf dem hier etwa $1,2 \mathrm{~mm}$ in der Quere messenden Mundkegel und vom Vorderrand fast $1 \mathrm{~mm}$ entfernt. Der Mundsaugnapf ist bald mehr kuglig, bald mehr in die Länge gestreckt; sein Längsdurchmesser beträgt $0,31-0,36 \mathrm{~mm}$, der quere $0,27-$ $\left.0,33 \mathrm{~mm}^{1}\right)$.

Der Bauchsaugnapf erscheint von der Fläche gesehen meist kreisrund, sein Eingang ist länglich oder dreieckig mit nach vorn gerichteter Basis; Quer- und Längsdurchmesser des ganzen Organs schwanken um etwa $1,4 \mathrm{~mm}$ herum $(1,3-1,56 \mathrm{~mm})$, die Wand selbst ist $0,3 \mathrm{~mm}$ und die ihn umgebende äussere Cuticula $0,021 \mathrm{~mm}$ dick.

Vom Oesophagus habe ich immer nur die wie ein Pharynx erscheinende Einmündung an der Gabelstelle des Darmes gesehen; von da ziehen die Darmschenkel zuerst in querer oder etwas nach vorn sehender Richtung seitwärts und biegen dann scharf nach hinten um; sie ziehen ein wenig divergirend bis zum Bauchsaugnapf, passiren an diesem rechts und links im Bogen vorbei und treten dann in den Hinterkörper über. Hier nähern sie sich und verlaufen nun, grössten Theils von den Dotterstocksfollikeln verdeckt, jedoch unmittelbar an den Seitenrändern des Mittelfeldes nach hinten; meist treten sie zwischen den beiden Hoden auf eine kurze Strecke deutlicher hervor (Fig. 1); hinter dem hintern Hoden nähern sie sich erheblich. Bei einem der 3 Exemplare aus Genua waren sie mit einer braunen Masse (Blut) ganz gefüllt und konnten ohne jede Schwierigkeit verfolgt werden; hierbei stellte es sich nun heraus, dass sie im Hinterleibe an ihrer Aussenseite mit längern und kürzern schlauchförmigen Blindsäcken besetzt sind, die sich gelegentlich gabeln und seitlich sowie nach hinten so weit wie die Dotterstocksfollikel erstrecken (Fig. 3). Dieses Verhalten des Darms ist für Clin. heterostomum charakteristisch, keine der übrigen! von Andern oder mir untersuchten Arten weist nach aussen gerichtete Blindschläuche an den Darmschenkeln auf, und bei

1) Bei allen diesen Maassen ist nie die Lichtung, sondern das ganze Organ von Aussenrand zu Aussenrand in der Längs- resp. Querrichtung des Thieres gemessen. 
keiner Art ziehen letztere so nahe den Seitenrändern des Mittelfeldes wie hier. Nachdem ich auf diese Anhänge einmal aufmerksam geworden war, gelang es auch, sie bei andern Exemplaren verschiedner Herkunft, wo sie entweder gar nicht oder nur in einzelnen ganz kurzen Abschnitten gefüllt waren, zwischen den Dotterstocksfollikeln aufzufinden; namentlich leicht ist das am Aussenrande des Dotterstocks, wo die Follikel nicht so dicht stehen oder kleine Längsstrassen zwischen sich frei lassen (Fig. 1).

Während nun die Darmschenkel der übrigen Clinostomen gewöhnlich mit ganz kleinen Blindsäcken besetzt sind, finden wir solche bei Clin. heterostomum in schwächerer Ausbildung nur an den Blindschläuchen.

Vom Excretionssystem habe ich bei dieser Art nichts wahrnehmen können.

Im Halstheil erscheinen zahlreiche kleine Gebilde, die ich für D r üsen halten möchte; Näheres hierüber bei einer andern Art.

Die Genitalien liegen nur im Hinterleibe, und zwar finden wir den Genitalporus ziemlich genau in der Mitte der Mittellinie, so dass demnach das Genitaldrüsenfeld hinter der Mitte des Hinterleibes gelegen ist. Der vordere Hoden erscheint von der Fläche gesehen hufeisenförmig mit nach vorn sehender Concavität, der hintere ist ausgesprochen herzförmig mit nach hinten gerichteter Spitze. Zwischen beiden Hoden liegt rechts der bei dieser Art verhältnissmässig grosse, $0,5 \mathrm{~mm}$ im Querdurchmesser haltende Keimstock, links davon die Schalendrüse mit dem Anfangstheil des Uterus und in der Mitte des Vorderrandes des hintern Hodens das Dotterreservoir. Clin. heterostomum ist jene Species, bei welcher der Uterus in so fern primitivere Verhältnisse zeigt, als der aufsteigende Schenkel am linken Seitenrande des Mittelfeldes nach vorn zu bis zum Bauchsaugnapf sich fortsetzt und hier bogenförmig in den zum Uterussack erweiterten absteigenden Schenkel übergeht (Fig. 1 u. 2). Hier häufen sich die braunschaligen Eier bei manchen Exemplaren sehr an; ihr Längsdurchmesser beträgt $0,125-0,135 \mathrm{~mm}$, der quere $0,062-0,073 \mathrm{~mm}$.

Der Genitalporus ist ein quer gestellter Spalt $(0,062 \mathrm{~mm}$ in der Quere); in seiner unmittelbaren Nähe ist der verhältnissmässig kleine Cirrusbeutel leicht zu bemerken. Die ganzen Seitenfelder des Hinterleibes werden bis auf eine schmale Randzone von den grossen Dotterstocksfollikeln eingenommen, welche die Darmschenkel mit ihren Blindschläuchen mehr oder weniger verdecken.

Wirthe und Fundorte sind schon oben angegeben. 


\section{Clinostomum complanatum (RUD.).}

(Taf. 1, Fig. 6 u. 7.)

1819. Distoma complanatum Rudolphi, Synops., p. 98 u. 376.

1845. Distoma complanatum Dujardin, Hist. nat. helm., p. 399.

1850. Distomum complanatum Diesing, Syst. helm., V. 1, p. 338.

1887. Dist. complanatum (=D. hians) Parona, in: Ann. Mus. civ, Genova (ser. 2) V. 4, p. 489.

1899. Clinostomum complanatum Braun, in: Zool. Anz., V. 22, p. 490.

1900. Clinostomum complanatum Braun, in: Ctrbl. Bakt., V. 27, Abth. 1, p. 27.

Das mir zur Verfügung stehende Material waren 4 Exemplare aus der Berliner Sammlung mit der Bezeichnung ,D. complanatum Rud. No. 1460. Ardea cinerea. Coll. Rud. Rosenthal S.", die Typen der Art, ferner 2 Exemplare aus der Genueser Sammlung mit der Bezeichnung: „Dist. complanatum (= D. hians), Ardea cinerea, Genova 10./4. $1886^{\prime \prime}$.

Ich beschreibe zuerst die Originalexemplare, welche durch die Einwirkung des aus den Korken extrahirten Tannins stark gebräunt sind, aber nach Aufhellung in Creosot die innern Organe erkennen lassen. Die Thiere (Fig. 6) sind langgestreckt elliptisch, das Vorderende abgestutzt, das hintere abgerundet; ihre Länge beträgt 3,5 bis 4,3 mm; Bauchfläche eben, Rückenfläche ziemlich stark gewölbt; Hals kurz, nur den 5.-6. Theil der Gesammtlänge betragend; seine hintere Grenze fällt mit dem Vorderrande des Bauchsaugnapfs zusammen, da sich hier eine Einziehung an den Seitenrändern findet; der Hals ist nur unerheblich schmäler als der Hinterleib.

Das Mundfeld hat quer ovale Gestalt und ist bei allen Exemplaren ziemlich eben, doch ventralwärts gerichtet; der Mundsaugnapf misst 0,29 in der Quer-, 0,16 mm in der Längsrichtung des 'Thieres.

Im Halstheil sind seitlich zwei dunkle, granulirte Längsstreifen zu erkennen, welche den Anfangstheil der Darmschenkel decken; eine ähnlich granulirte Masse liegt zwischen den Darmschenkeln — alle diese Granula halte ich für einzellige Drüsen. Die Darmschenkel selbst sind, soweit erkennbar, mit ganz kleinen Ausbuchtungen versehen; sie verlaufen ungefähr parallel den Körperränderu, etwa in der mittlern Partie der Seitenfelder, also nicht an der Grenze dieser gegen das Mittelfeld, und convergiren hinten bis zur Berührung der blinden Enden.

Der kräftige Bauch saug n apf ist nicht ganz kreisrund, sondern mehr dreieckig wie auch seine Mündung; Quer- wie Längsdurchmesser betragen $0,5 \mathrm{~mm}$. 
Das Genitaldrüsenfeld liegt in der Mitte des Hinterleibes; die beiden Hoden sind nicht gleich gross, doch von ähnlicher dreieckiger Gestalt; durch zwei seitliche Einschnitte grenzt sich an dem hintern Hoden ein mittlerer nach hinten gerichteter Lappen deutlicher ab, am vordern Hoden ein vorderer Lappen; die einander zugekehrten Flächen der Hoden sind leicht ausgehöhlt.

Zwischen ihnen liegen rechts der kuglige, 0,19 mm im Durchmesser haltende Keimstock, ferner in der Mitte und links der Anfangstheil des Uterus, umgeben von Schalendrüsenzellen, und in der Mitte des Hinterrandes des hintern Hodens das Dotterreservoir, in welches die queren Dottergänge einmünden.

Rechts neben dem vordern Hoden und vor dem Keimstock liegt der Cirrusbeutel, der eine kleine Vesicula seminalis umschliesst, ebenfalls rechts von der Mittellinie des Genitalporus ${ }^{1}$ ); ich glaube mich davon überzeugt zu haben, dass der links am Vorderhoden nach vorn ziehende Uterus von der linken Seite in den Uterussack mündet, der sich über diese Stelle hinaus bis zum Hinterrand des Bauchsaugnapfs erstreckt. Die Eier sind braun, ziemlich bauchig, 0,12 mm lang, $0,07 \mathrm{~mm}$ breit.

Mit Ausnahme eines schmalen Seitenstreifens sowie des Hinterendes nehmen die Dotterstocksfollikel die ganzen Seitenfelder ein; hinter den Hoden liegen sie auch im Mittelfeld, doch bleibt das Hinterende so weit frei, dass hier die letzten Enden der Darmschenkel nicht von Follikeln bedeckt sind.

Die beiden Genueser, ebenfalls aus Ardea cinerea stammenden Exemplare verhielten sich verschieden; das eine war stark contrahirt, 6,1 $\mathrm{mm}$ lang, im Hals 1,1, im Hinterleib 2,6 $\mathrm{mm}$ breit; das andere war offenbar unter starkem Druck des Deckglases abgetötet worden, 9,5 mm lang und ganz abgeplattet (Fig. 7); beide Exemplare eigneten sich sehr gut für die weitere Untersuchung nach Aufhellung in Creosot resp. Glycerin. Sie erwiesen sich mit den Typen von Dist. complanatum RuD. trotz einzelner Differenzen so sehr übereinstimmend, dass ich sie unbedenklich für dieselbe Species halte.

Die Uebereinstimmung lag in der Kürze des Halstheiles, in der geringen Differenz zwischen dessen und der Breite des Hinterleibes, in der Lage des Genitaldrüsenfeldes, des Cirrusbeutels, des Genital-

1) Die Angabe von MacCallum (1. c.), dass Dist. complanatum Rud. den Genitalporus vor dem Bauchsaugnapf liegen habe, ist unrichtig; Rudolpur giebt hierüber nichts bestimmtes an. 
porus, der Ausdehnung der Dotterstöcke, dem Verhalten der Darmschenkel und des Vorderendes; auch die Eier wiesen fast dieselben Grössenverhältnisse auf $(0,104: 0,062 \mathrm{~mm})$. Eine Differenz bestand nur in dem Aussehen der Hoden, da der Vorderrand des vordern und der Hinterrand des hintern Hodens bei dem gestreckten Exemplar eine grössere Zahl von Einschnitten zeigten, so dass mehr Lappen gezählt werden konnten, während bei dem contrahirten Exemplar meinen Notizen nach — die vordern resp. hintern Ränder der beiden Hoden tiefer als bei den Typen eingeschnitten waren; es mag also sein, dass auch bei letztern die Hoden deutlicher gelappt sind, als sie mir erschienen, und dass bei Abflachung des Körpers noch weitere Einkerbungen deutlich werden. Jeden Falls ist auch bei dem gestreckten Exemplar der vordere Hoden etwas kleiner und weniger zahlreich gelappt als der hintere. Ferner ist bei dem contrahirten Genueser Exemplar - von dem platt gedrückten muss abgesehen werden - der Bauchsaugnapf nicht unerheblich grösser, 0,7 resp. 0,8 gegen 0,5 mm bei dem Typus. Diese Differenzen genügen jedoch lange nicht zur Abzweigung einer besondern Art.

Höchst wahrscheinlich gehören die von v. Linstow aus Nycticorax griseus beschriebenen Exemplare (D. heterostomum) zu Clinostomum complanatum (RUD.).

\section{Clinostomum marginatum (RuD.).}

(Taf. 1, Fig. 4, 8; Taf. 2, Fig. 19, 20.)

1819. Distoma marginatum Rudolphi, Synops., p. 680.

1845. Distoma marginatum Dujardin, Hist. nat. helm., p. 446.

1850. Distomum dimorphum p. p. Diesing, Syst. helm., V. 1, p. 354. 1856. Distomum dimorphum p. p. Diesing, in: Denkschr. Akad. Wiss. Wien, math.-nat. Cl., V. 10, p. 65.

1899. Clinostomum marginatum Braun, in: Zool. Anz., V. 22, p. 490. 1900. Clinostomum marginatum Braun, in: Ctrbl. Bakt., V. 27, Abth. 1, p. 28.

Auch von dieser Art will ich zuerst die Typen beschreiben, welche (6 Exemplare) in der Berliner Sammlung erhalten sind und die Aufschrift führen: ,Dist. marginatum Rud.;?= Dist. dimorphum Dies. var.? Ardea sp. Brasilien; v. Olfers. Coll. Rud. No. 1493“.

Die Thiere sind bis $7 \mathrm{~mm}$ lang (Fig. 4), ihre Bauchfläche ist eben, die Rückenfläche nur sanft gewölbt; die geringere Dicke und eine leichte Quellung der Exemplare bedingt wohl ihre gegenüber Clin. complanatum bemerkenswerthe grössere Durchsichtigkeit, so dass die Anwendung von Glycerin zur Aufhellung völlig ausreichte. 
Das Mundfeld war mehr oder weniger vorgewölbt, so dass das Vorderende abgerundet erschien; der Hals nimmt etwa ein Viertel der Gesammtlänge in Anspruch bei einer Breite von 1,2 mm, die grösste Breite des Hinterleibes betrug $2 \mathrm{~mm}$. Der Mundsaugnapf ist kuglig, sein Quer- wie Längsdurchmesser beträgt $0,28 \mathrm{~mm}$; der Bauchsaugnapf ist fast dreimal so gross $(0,75 \mathrm{~mm})$, dickwandig $(0,24 \mathrm{~mm})$ und hat eine mehr oder weniger deutlich dreieckige Mündung.

Im Halstheil, zwischen der Begrenzung des Mundfeldes und dem Bauchsaugnapf, sind auch hier sehr zahlreiche, kuglige Körperchen $\mathrm{zu}$ erkennen (Drüsen?).

Die Darmschenkel zeigen in ihrem ganzen Verlauf, von der Umbiegungsstelle nach hinten bis zum blinden Ende kleine Aussackungen; sie verlaufen vorn etwas divergirend, nähern sich hinter dem Bauchsaugnapf etwas und ziehen dann ungefähr den Seitenrändern des Hinterleibes parallel nach hinten, wo sie sich mit den blinden Enden einander zuneigen.

Das Genitaldrüsenfeld liegt hinter der Mitte des Hinterleibes; die Hoden sind ungleich gross, der kleinere, vordere etwas nach links verschoben, der grössere, hintere liegt dagegen symmetrisch zur Medianebene; im Ganzen erscheinen sie von der Fläche gesehen als dreiseitige Gebilde mit abgerundeten Ecken, deren einander zugekehrte Flächen ein wenig vertieft sind; die vordern resp. hintern Seiten sind nicht geradlinig, sondern schwächer oder tiefer gekerbt oder selbst eingeschnitten, so dass sich am hintern Hoden ein nach hinten gerichteter medianer Lappen mitunter deutlich abgrenzt; in einem Fall war auch er durch eine tiefe Einkerbung gespalten; weniger deutlich ist je eine Einkerbung an den Vorderrändern des vordern Hodens, der überhaupt eine asymmetrische Gestalt besitzt.

Der Genitalporus liegt fast genau in der Mittellinie, also nicht rechts wie bei Clin. complanatum; rechts vom rechten Vorderrande des Hodens finden wir den Cirrusbeutel, dessen Cirrus bei manchen Exemplaren aus dem Porus hervorsah. Der Keimstock ist kuglig oder ganz seicht an der Peripherie eingekerbt; er liegt wie gewöhnlich rechts, zwischen dem Hinterende des Cirrusbeutels und der rechten Seitenspitze des hintern Hodens; in der Mitte des Vorderrandes des letztern bemerkt man wieder das Dotterreservoir mit den queren Dottergängen. Davor liegt der Anfangstheil des Uterus, der hier zwei quer gestreckte Schlingen bildet und dann am Hinterrande des vordern Hodens entlang nach links zieht, um neben dem Hoden nach vorn und in die Mitte zu gelangen, wo er in den Uterussack 
einmündet. Nie war letzterer ganz gefüllt, er erschien vielmehr immer als ein schmaler, bis an den Bauchsaugnapf reichender und hier blind endigender Kanal, dessen Wandungen sich in der hintern Partie erheblich verdickten, so dass eine spindelförmige Auftreibung deutlich hervortrat; in ihm befanden sich immer nur wenige Eier, jeden Falls weit weniger als in dem übrigen Uterus. Die Eier sind ziemlich dünnschalig, gelblichbraun, elliptisch, 0,14 mm lang, 0,073 mm breit.

Eine sehr starke Ausbildung haben die Dotterstöcke erfahren; ihre Follikel lassen im Hinterleib nur je einen schmalen Seitenstreifen, das äusserste Hinterende, das Genitaldrüsenfeld und den schmalen schlauchförmigen Uterussack frei; ob sich jemals der letztere stärker füllt und ob dann die Follikel mehr seitwärts verschoben werden, lässt sich an dem vorliegenden Material nicht entscheiden.

Wie ich in meinen vorläufigen Mittheilungen schon angegeben habe, finden sich unter den mir von der Direktion des K. K. naturhistorischen Hofmuseums in Wien anvertrauten Clinostomen, welche NATTERER auf seiner Reise in Brasilien gesammelt hat, auch Formen, deren Zugehörigkeit zu Dist. marginatum Rud. schon Diesing richtig erkannt hat. Es sind folgende:

1) Glas No. 831. X. 644 mit der Aufschrift: „Distoma; oes. Ardea Heron couleur de plomb" (=Ardea cocoi). Hier finden sich zahlreiche kleinere Exemplare von 4-5 mm Länge, die durchweg auch schlanker sind, wie die RudoLPH'schen Typen der Art; einige sind noch nicht geschlechtsreif und enthalten keine Eier (Fig. 19), andere zeigen den Uterussack prall mit Eiern gefüllt (Fig. 20), so dass dieser einen schon mit blossem Auge erkennbaren braunen Fleck hinter dem Bauchsaugnapf darstellt, noch andere führen nur wenige Eier im Uterussack (Fig. 8). Bei den unreifen Exemplaren war der Uterussack deutlich erkennbar; er stellte einen kurzen vom Genitalporus bis fast an den Bauchsaugnapf reichenden Schlauch von lang gestreckter Gestalt, aber grösserer Breite als bei den RudoLPH'schen Typen dar; auch war eine spindelförmige Auftreibung an seinem Hinterende nicht erkennbar. Die Dotterstöcke verhalten sich wie bei den Typen, nur bei prall gefülltem Uterussack waren sie aus dem vordern Theil des Mittelfeldes nach den Seiten verdrängt. Das Genitaldrüsenfeld liegt in der Mitte des Hinterleibes, der Genitalporus in der Mittellinie dicht vor dem vordern Hoden; rechts von diesem der Cirrusbeutel, hinter diesem wiederum der meist kuglige Keimstock. Die Hoden sind quer gestreckte Organe, weniger ungleich als bei den Typen, der 
vordere am vordern, der hintere am hintern Rande eingekerbt, der hintere meist nur mit 2 Einkerbungen, so dass sich ein unpaarer hinterer Lappen deutlich abgrenzte.

Das Mundfeld zeigte die typische Form, welche bei den Originalexemplaren nicht hervortrat, da sich das ganze Vorderende vorgestreckt hatte, hier dagegen trat Ringfurche und Mundkegel deutlich hervor. Der Mundsaugnapf war meist kuglig oder etwas in die Quere gestreckt; sein Durchmesser betrug ungefähr 0,25 mm; dreimal so gross ist der Bauchsaugnapf, der gewöhnlich eine dreieckige Mündung aufwies; die Wanddicke betrug $0,23 \mathrm{~mm}$. Die Darmschenkel verhielten sich wie bei den Typen. Bei unreifen Exemplaren war das oberflächliche Gefässnetz meist in grössern Abschnitten erkennbar (Fig. 19).

In demselben Glase befand sich endlich noch ein $8 \mathrm{~mm}$ langes Exemplar, das in der Körpergestalt dem Dist. heterostomum RuD. ähnelte, in der innern Organisation aber fast vollständig mit Dist. marginatum RuD. übereinstimmte - selbst in der Form der Hoden; jedoch sind die Saugnäpfe grösser (Mundsaugnapf quer 0,37, längs $0,3 \mathrm{~mm}$, Bauchsaugnapf quer 1,07, längs $0,97 \mathrm{~mm})$, die Breite des Halses beträgt 1,5 , die des Hinterleibes $2,3 \mathrm{~mm}$.

Die Maasse für die ziemlich dünnschaligen Eier schwankten bei allen diesen Exemplaren zwischen 0,104-0,114 mm in der Länge und $0,055-0,064$ in der Breite.

2) Glas No. 750. VIII. 350; Aufschrift: „Distoma detruncatum D Mycteria americana". Neben zahlreichen Exemplaren von Clinostomum detruncatum findet sich ein kleines Exemplar, das mit Dist marginatum RUD. übereinstimmt; die Hoden sind nicht so stark wie bei den Exemplaren aus Glas 831, in die Quere gestreckt, und der Keimstock erscheint von der Fläche gesehen nicht kreis- sondern nierenförmig.

3) Glas No. 342. 906; Aufschrift: „50b Dist. marginatum Ardeae"; 6 Exemplare, 3 von ihnen etwas verletzt; auch sie stimmen gut zu den Rudolphi'schen Typen von Dist. marginatum; ihre Länge schwankt zwischen 4,5 und $6 \mathrm{~mm}$; der Halstheil ist ziemlich schmal; das Genitaldrüsenfeld liegt dicht hinter der Mitte des Hinterleibes, der mit Ausnahme des vordern Theiles des Mittelfeldes, wo der lang gestreckte und schmale Uterussack liegt, ganz mit dicht stehenden Dotterstocksfollikeln besetzt ist. Der Mundsaugnapf ist ein wenig in die Quere gestreckt $(0,21-0,26$ quer, 0,20 mm lang), der Bauchsaugnapf etwa dreimal so gross $(0,57-0,62 \mathrm{~mm})$; seine Mündung ist drei- 
eckig mit nach vorn gerichteter Basis. Das Mundfeld erweist sich von dem gewöhnlichen Aussehen; Eier 0,12-0,14 mm lang, 0,073 $-0,075 \mathrm{~mm}$ breit.

Wie gesagt hege ich keinen Zweifel, dass die hier erwähnten, von NATTERER gesammelten Clinostomen nicht nur unter einander, sondern auch mit dem RudolPHI'schen Distomum marginatum übereinstimmen; ein durchgreifendes unterscheidendes Merkmal wüsste ich nicht anzuführen; dass bei einigen das Genitaldrüsenfeld in der Mitte des Hinterleibes, bei andern unmittelbar hinter dieser liegt, kann sehr wohl von der Contraction abhängen; auch die Form der Hoden ist nicht so wesentlich verschieden, sie wird sicherlich beeinflusst durch den jeweiligen Entwicklungszustand, in welchem sich das Organ befindet, sowie durch den Erhaltungszustand des betreffenden Exemplars. In dieser Beziehung habe ich schon angeführt, dass die Typen des Dist. marginatum gequollen erscheinen.

Weiterhin aber unterliegt es mir keinem Zweifel mehr, dass auch die von Ramsay Wright und von MacCallum als ,? Distomum heterostomum" resp. "Clinostomum heterostomum" beschriebenen Formen zu Unrecht diesen Namen tragen: sie sind Dist. marginatum Rud., ich wüsste wenigstens keine Art zu nennen, mit der diese frisch untersuchten Exemplare besser übereinstimmten; kleine Differenzen blèiben auch hier bestehen, wie z. B. die Grösse der Eier, welche von beiden Autoren mit 0,099 resp. 0,066 mm angegeben wird, also (namentlich in der Länge) geringer ist als die von mir gefundene; auch die Saugnäpfe giebt $R$. WRIGHT grösser an (Mundsaugnapf $0,3 \mathrm{~mm}$, Bauchsaugnapf $0,8 \mathrm{~mm}$ ) - dem gegenüber stehen so viele Uebereinstimmungen, dass eine Trennung unmöglich ist.

Nun fragt es sich aber, ob Dist. complanatum Rud. nicht mit Dist. marginatum RuD. identisch ist; beide Arten sind gewiss recht ähnlich, dass sie RudoLPHI trotzdem nicht identificirt hat, dürfte einmal in dem Umstande liegen, dass die eine Art europäischer, die andere südamerikanischer Herkunft ist, sodann aber auch in dem verschiedenen Aussehen der noch vorhandenen Typen: D. complanatum ist ziemlich kurz und gedrungen, nicht so breit und nicht so abgeflacht wie D. marginatum. An diesen äussern Merkmalen sind die conservirten Exemplare beider Arten sofort aus einander zu halten; bestehen daneben aber auch Unterschiede in der Organisation? Ich glaube solche anführen zu können: 1) ist der Hals des $D$. complanatum recht kurz und der Bauchsaugnapf sehr dem Vorderende genähert; 2) liegt der Genitalporus nicht in der Mittellinie, sondern erheblich rechts von 
M. BRAUN,

dieser; 3) liegt die vordere Grenze der Dotterstöcke bei D. complanatum etwas weiter nach hinten, ebenso hören dieselben hinten etwas früher auf; weniger Gewicht dürfte darauf zu legen sein, dass das Genitaldrüsenfeld bei D. complanatum in der Mitte des Hinterleibes liegt. Im Uebrigen erweisen sich allerdings beide Arten als sehr ähnlich; berücksichtigt man aber auch noch die verschiedene Körpergestalt und das Vaterland ${ }^{1}$ ), so dürfte die Trennung beider Formen, die man als vicariirende Arten ansehen kann, sich rechtfertigen lassen.

Wie man aber auch diese Frage beantworten mag, so viel steht fest, dass die nordamerikanische, bisher zu Dist. heterostomum gestellte Art nur Dist. marginatum sein kann.

\section{Clinostomum foliiforme BRAUN.}

(Taf. 1, Fig. 5.)

1896. Distomum (Dicrocoelium) heterostomum Parona, in: Boll. Mus. Zool. Anat. comp. Torino, V. 11, 1896, No. 258.

1899. Clinostomum foliiforme BraUn, in: Zool. Anz., V. 22, p. 490.

1899. Clinostomum foliiforme Braun, in: Boll. Mus. Zool. Anat. comp. Torino, V. 14, No. 364.

1900. Clinostomum foliiforme Braun, in: Ctrbl. Bakt., V. 27, Abth. 1, p. 31.

Vor wenigen Jahren revidirte C. PARONA die Helminthensaminlung des Museums für Zoologie und vergleichende Anatomie in Turin; in der hierüber publicirten Mittheilung $(1896,1$. c.) erwähnt Parona, Dist. (Dicrocoelium) heterostomum Rud. in der Turiner Sammlung in 2 grossen und 1 kleinern Exemplar gefunden zu haben. Auf meine Bitte übersandte mir Herr College Camerano 2 Fläschchen mit Trematoden, welche beide die Aufschrift: Dicrocoelium heterostomum RuD. (aus Ardea purpurea) tragen; das eine Glas mit der Nummer „C. N. 359“ enthielt die von Parona (l. c.) angeführten Exemplare, die beiden grössern sind in der That Dist. heterostomum RuD., das kleinere jedoch eine andere Art, die sich in 10 Exemplaren in dem zweiten Fläschchen (C. N. 361) befindet; diesen Fund hat PARONA nicht besonders erwähnt, wohl aber das Glas mit der Aufschrift: „Dicrocoelium heterostomum Rud.“ versehen; ausserdem trug dasselbe noch eine von A. DE FiLipPI herrührende Etiquette: „Distoma heterostomum RuD. - Ardea purpurea, pharynx et oesoph." Zu dieser

1) Man vergl. in dieser Beziehung die Bemerkungen von A. Looss, in: Zool. Jahrb., V. 12, Syst., 1899, p. 607, Anm. 
Form gehören dann endlich noch 2 Exemplare, welche am 12. Mai 1879 in einer zu Genua erlegten Ardea purpurea gesammelt sind, in der Sammlung des Zoologischen Instituts zu Genua aufbewahrt werden und mir zur Untersuchung von Herrn Collegen PARONa übersandt wurden.

Meine Beschreibung stützt sich auf die Untersuchung der 10 Exemplare aus der Turiner Sammlung; die übrigen 3 weichen von ihnen nicht ab.

Clinostomum foliiforme ist eine recht kleine Art, die im conservirten Zustande 3,6, 4,0 resp. 4,6 mm lang ist; der Körper ist ganz flach, auf der Bauchfläche mehr oder weniger ausgehöhlt und dem entsprechend dorsal gekrümmt - dieses Verhalten ist aber jeden Falls nur eine Folge der Conservirung, der Dickendurchmesser bleibt überall derselbe und stets sehr klein. Die Thiere fühlen sich trotz ihrer Kleinheit derb an und sind gelblich durchscheinend.

Bemerkenswerth ist ferner der Umriss des Körpers; von dem abgerundeten aber schmalen Vorderende ziehen die seitlichen Begrenzungen divergirend nach hinten; die grösste Breite $(1,33-1,76 \mathrm{~mm})$ liegt vor dem Genitaldrüsenfeld, von da verschmälert sich der Körper wieder nach hinten. Der Halstheil dieser Art setzt sich demnach nicht so scharf vom Hinterkörper ab, wie bei den bisher beschriebenen Arten, er ist conisch und geht allmählich in den Hinterkörper über. Das Mundfeld erscheint mehr längs oval oder kreisförmig, die Ringfurche ist bei der geringen Körperdicke nur seicht oder ganz verstrichen; vor ihrer Mitte liegt die Mundöffnung. Der Mundsaugnapf ist meist etwas länger als breit, die Maasse schwanken zwischen 0,25 und $0,3 \mathrm{~mm}$; etwa $3 \mathrm{mal}$ so gross ist der ungemein dickwandige Bauchsaugnapf $(0,75-0,833 \mathrm{~mm})$, dessen Eingang gewöhnlich schlitzförmig ist, bei tieferer Einstellung des Mikroskops ist der optische Schnitt der Saugnapfhöhlung herzförmig oder dreieckig.

Die Darmschenkel sind vom Bauchsaugnapf an mit kleinen Blindsäckchen dicht besetzt; sie reichen hinten über die Dotterstöcke hinaus.

Das Genitaldrüsenfeld fällt in die Mitte des Hinterleibes; die beiden Hoden sind 3 lappig, in die Quere gestreckt und der vordere etwas grösser als der hintere; bei beiden Organen besteht die Tendenz zu weiterer Lappung: so kann am Vorderrande des hintern Hodens rechts und links von der Mittellinie je ein Einschnitt auftreten, wodurch sich auch ein vorderer medianer Lappen abgrenzt; auch am vordern Hoden tritt dies auf, aber an seinem Hinterrande, 
und wenn dann noch an der rechten und linken Vorderseite statt eines Einschnittes je zwei auftreten, so weist der Vorderhoden 6 Lappen auf: einen kleinen medianen vorn, einen breitern medianen hinten und je 2 Lappen an den Seiten; in solchem Fall sind dann Längsund Querdurchmesser des vordern Hodens gleich oder der erstere sogar etwas grösser. Man sieht, die Art der Lappenbildung an den Hoden dieser Art ist eine andere als bei Clinostomum marginatum, wo die einander zugekehrten Ränder der Hoden nicht eingekerbt werden, sondern nur die nach vorn resp. hinten gerichteten Begrenzungen; auch bleiben trotz aller Zerschlitzung dieser Ränder die Hoden mehr in der Querrichtung gestreckt.

Der Keimstock liegt an der gewöhnlichen Stelle; mitunter ist er zum Theil von Dotterstocksfollikeln bedeckt; seine Form ist kuglig, gelegentlich scheint es, als ob er sich mehr nach der Mittellinie zu ausdehnt. Auf derselben Seite findet sich vor dem Keimstock der Cirrusbeutel, und ebenfalls rechts, an dem rechten Seitenlappen des vordern Hodens, liegt der Genitalporus. Das in der Mittellinie zwischen den beiden Hoden gelegene Dotterreservoir ist verhältnissmässig gross und kuglig. Die grossen quer gestreckten Dotterstocksfollikel beginnen dicht hinter dem Bauchsaugnapf, nehmen die Seitenfelder und hinter den Hoden auch das Mittelfeld ein, lassen jedoch das hinterste Körperende frei. Die in der Abbildung angedeutete radiäre Richtung der Dotterstocksfollikel scheint ein weiteres Merkmal für Clinostomum foliiforme zu sein; sie trat auch bei andern Exemplaren mehr oder weniger deutlich hervor.

Der Uterus zeigt das gewöhnliche Verhalten, er beginnt also zwischen den Hoden, macht hier einige Schlingen, umzieht links im Bogen den vordern Hoden und geht vor diesem in den Uterussack über, dessen hinteres Ende nach rechts, dem Genitalporus zu gewendet ist. Die Eier sind nicht besonders zahlreich, aber recht gross: 0,135$0,145 \mathrm{~mm}$ lang, 0,065̃ $-0,729 \mathrm{~mm}$ breit.

\section{Clinostomum detruncatum BRAUN.}

(Taf. 2, Fig. 11.)

1850. Distomum dimorphum Diesing p. p., Syst. helm., V. 1, p. 354. 1856. Distomum dimorphum Diesing p. p., in: Denkschr. Akad. Wiss. Wien, math.-nat. Cl., V. 10, p. 65.

1899. Clinostomum detruncatum Braun, in: Zool. Anz., V. 22, p. 490. 1900. Clinostomum detruncatum Braun, in: Ctrbl. Bakt., V. 27, Abth. 1, p. 29. 
Unter den von Diesing zu Distomum dimorphum vereinten Clinostomen lassen sich mehrere Arten leicht aus einander halten, die ursprünglich zum Theil wenigstens auch Diesing trennte, wie die noch vorhandenen Originaletiketten beweisen; eine solche Form bezeichnet er handschriftlich mit $D$. detruncatum, ersetzte aber später selbst den Speciesnamen durch „dimorphum". Freilich sind auch hier wiederum 2 Arten vereint, die sich äusserlich sehr ähnlich sehen; ich behalte für die eine Art den ursprünglichen Speciesnamen bei, die andere nenne ich Clin. sorbens.

Clin. detruncatum ist in der Wiener Sammlung unter dem von Natterer gesammelten und von Diesing durchgearbeiteten Material in 2 Gläsern vertreten; das eine (No. 750. VIII. 350) enthält zahlreiche grössere und kleinere Exemplare aus Mycteria americana, das andere (No. 882. IX. 466) nur 2 aus dem Oesophagus von „Ardea maguari" $=$ Ciconia americana.

Die Art gehört zu den grössern, da sie bis $14 \mathrm{~mm}$ lang und $3 \mathrm{~mm}$ breit wird, das kleinste Exemplar ist allerdings nur $6 \mathrm{~mm}$ lang und $2 \mathrm{~mm}$ breit; die Bauchfläche ist eben oder etwas ausgehöhlt, die Rückenfläche gewölbt, der Dickendurchmesser zwar grösser als bei den bisher besprochenen Arten, aber nicht sehr erheblich. Vorderund Hinterende sind abgestutzt, und die Seitenränder des Körpers verlaufen ziemlich parallel, nur vorn und hinten neigen sie sich einander zu. Der Halstheil ist kurz, kaum schmäler als der Hinterleib, jedoch von diesem durch eine Einziehung an den Seitenrändern getrennt.

Recht kräftig sind die schon mit blossem Auge erkennbaren Haftorgane; das quer ovale und fast terminal stehende Mundfeld erreicht $1,3-1,7 \mathrm{~mm}$ im Querdurchmesser, der Mundkegel in derselben Richtung $0,7-1,0 \mathrm{~mm}$, die ebenfalls quer gestellte Mundöffnung meist nur $0,2 \mathrm{~mm}$ und der Mundsaugnapf 0,3-0,4 mm. Der Bauchsaugnapf, der dem Mundfeld sehr genähert ist, erscheint von der Fläche gesehen kreisförmig mit einem Durchmesser von $1-1,5 \mathrm{~mm}$, seine Wand ist sehr dick, der Eingang dreieckig mit nach innen eingebogenen Seiten.

Die Darmschenkel ziehen durch den ganzen Körper parallel den Seitenrändern bis ins äusserste Hinterende, wo sie sich im Bogen der Mittellinie nähern; auch sie sind wenigstens im Hinterleibe mit Blindsäckchen besetzt, die jedoch nicht so dicht stehen wie bei den bisher beschriebenen Arten.

Auch einzelne Theile des Genitalapparats sind mit blossem Zool. Jahrb. XIV. Abth. f. Syst. 
Auge erkennbar; das Genitaldrüsenfeld liegt ganz im Hinterende, die in die Quere gestreckten Hoden, deren Querdurchmesser über $1 \mathrm{~mm}$ beträgt, sind unregelmässig gelappt, der hintere stärker als der vordere, und die einander zugekehrten Ränder zeigen eine bedeutendere Concavität, als sie bei andern Arten zu verzeichnen war. Zwischen den beiden Hoden liegt rechts der Keimstock, den übrigen Raum nimmt die Schalendrüse mit dem Anfangstheil des Uterus und das Dotterreservoir ein.

Am Vorderrande des vordern Hodens tritt in der Mittellinie der Genitalporus deutlich hervor; zu ihm zieht von rechts her im Bogen der Cirrusbeutel, dessen Hinterende nicht den Keimstock berührt, sondern von diesem durch den Hoden getrennt ist. Bei einem Exemplar konnten die beiden Vasa efferentia erkannt werden; sie entspringen von der ventralen Fläche der Hoden, das hintere beinahe in der Mitte, das vordere dagegen rechts in der Nähe des Vorderrandes; das hintere, längere kreuzt den Keimstock und den rechten Theil des vordern Hodens und vereint sich mit dem sehr kurzen vordern Vas deferens unmittelbar vor dem Hinterende des Cirrusbeutels; die Vesicula seminalis liegt wie bei allen Clinostomen im Cirrusbeutel.

Die Dotterstöcke bestehen aus zahlreichen kleinen Follikeln, welche vorn unmittelbar hinter und neben dem Bauchsaugnapf beginnen, breitere Streifen an den Seiten frei lassen, im Uebrigen aber bis dicht an das Mittelfeld herantreten; auch hinter dem hintern Hoden fehlen sie nicht.

Der zum Uterussack führende Gang verläuft links am vordern Hoden vorbei und mündet von links her in den hintern Theil des Sackes. Dieser erreicht eine Länge von $3-5 \mathrm{~mm}$, trotzdem aber nicht den Hinterrand des Bauchsaugnapfes. Bei jüngern Exemplaren stellt er einen einfachen, ziemlich weiten Schlauch dar, mit zunehmender Füllung durch Eier treibt dieser auf beiden Seiten kurze, mitunter sich gabelnde Sprossen, die dem Organ das Aussehen des Uterus einer Cystotaenie verleihen. DieEier sind 0,11 mm lang, 0,062 mm breit.

\section{Clinostomum sorbens BRAUN.}

(Taf. 2, Fig. 13, 14 u. 15.)

1850. Distomum dimorphum p. p. Diesing, Syst. helm., V. 1, p. 354. 1856. Distomum dimorphum p. p. Diesing, in: Denkschr. Akad. Wiss. Wien, math.-nat. Cl., V. 10 , p. 65.

1899. Clinostomum sorbens Braun, in: Zool. Anz., V. 22, p. 490. 
1900. Clinostomum sorbens Braun, in: Ctrbl. Bakt., V. 27, Abth. 1, p. 30 .

Diese Art ist in der Wiener Sammlung nur durch 5 Exemplare vertreten, welche Diesing wegen ihrer Aehnlichkeit in Form und Grösse mit Clinostomum detruncatum nicht von dieser Art unterschieden hatte; das betreffende Glas (No. 878. 804) trägt daher auch dieselbe Bezeichnung (Distoma detruncatum D.), die Exemplare stammen jedoch aus dem Oesophagus von Tantalus loculator.

Clinostomum sorbens bietet so zahlreiche Differenzen von den übrigen Clinostomen dar, dass man es sehr wohl zum Vertreter einer besondern Gattung machen kann.

Auch diese Art gehört zu den grössern und massigern; sie erreicht 9-10 $\mathrm{mm}$ Länge bei einer Breite von 2,5-2,9 $\mathrm{mm}$. Der Körper ist lang gestreckt, vorn und hinten etwas verjüngt, die Bauchfläche eben oder concav, die Rückenfläche gewölbt, der Dickendurchmesser beträchtlich (Fig. 15).

Das Vorderende weicht in so fern von dem anderer Clinostomen ab, als die den Mundkegel umziehende Ringfurche nicht vollständig um diesen herumzieht, sondern auf der Bauchseite rechts und links von der Mittellinie blind und erweitert endet (Fig. 15); ein Ausguss der Furche würde also nicht einem vollständigen Ring, sondern einer Armspange oder einem Hufeisen gleichen, dessen freie Enden verbreitert sind. Die quer ausgezogene Mundöffnung liegt subterminal auf dem Mundkegel; der Mundsaugnapf misst 0,350 mm in der Längs-, 0,469 $\mathrm{mm}$ in der Querrichtung (des Thieres).

Recht deutlich trat gewöhnlich das pharynxartige Ende des Oesophagus hervor; die Darmschenkel sind meist nur im Vorderende, das sich kaum irgendwie bezeichnend vom Hinterleibe absetzt, deutlich zu erkennen; immerhin konnte festgestellt werden, dass sie auch hier bis ins äusserste Hinterende ziehen und im Hinterleibe mit kurzen Ausbuchtungen besetzt sind.

Der Bauchsaugnapf, der 2-2,5 mm von der Mundöffnung entfernt ist, ist kräftig und dickwandig, sein Durchmesser beträgt $1-1,3 \mathrm{~mm}$.

Das Genitaldrüsenfeld nimmt wegen der starken Ausbildung der beiden Hoden die ganze hintere Hälfte des Hinterleibes ein. Die Gestalt der Hoden ist schwer zu beschreiben; den vordern könnte man bisquitförmig nennen, sein Querdurchmesser, welcher 1,4-1,6 mm beträgt, ist etwas grösser als der in die Längsaxe fallende, wogegen beim hintern Hoden die Längserstreckung die grössere ist; er ist von 
hinten her tief eingeschnitten, so dass nur eine schmale, dorsale Brücke die beiden Hälften vereinigt. Der Vorderrand beider Hoden ist vertieft und ihre ganze Oberfläche ist auf der Rücken- wie auf der Bauchseite nicht gleichmässig eben, wie es die Abbildungen zeigen, sondern mit grössern und kleinern Buckeln versehen.

Zwischen den beiden Hoden liegt rechts der kleine, mehr kuglige oder etwas gestreckte Keimstock, neben ihm, auch noch auf der rechten Seite, der ziemlich grosse Cirrusbeutel, dessen Cirrus bei einigen Exemplaren aus der etwa in der Mittellinie zwischen den Hoden gelegenen Geschlechtsöffnung hervorgestreckt ist.

Vor dem vordern Hoden findet man im Mittelfeld den länglich ovalen Uterussack (2,3 mm lang, 1,2 $\mathrm{nm}$ breit); allem Anschein nach zieht der zu ihm führende Uterusgang, der zwischen den Hoden beginnt, ventral vom vordern Hoden nach vorn; wahrscheinlich besteht ein zweiter ventraler Gang, der vom Uterussack zum Genitalporus führt. Die Eier sind 0,104-0,114 mm lang, 0,073-0,083 $\mathrm{mm}$ breit.

Auch die Dotterstöcke zeigen Abweichungen von dem gewöhnlichen Verhalten; 1) sind ihre Follikel nicht so sehr zahlreich, 2) nehmen sie kleinere Partien des Hinterleibes ein und 3) finden sich Dotterstocksfollikel schon vor dem Bauchsaugnapf. Die Follikel liegen, wie übrigens auch bei andern Clinostomen im Hinterleibe auf der Ventralund Dorsalfläche und bilden bei Clin. sorbens zwei nach der Medianebene offene Rinnen, in denen die Darmschenkel nach hinten verlaufen.

\section{\%. Clinostomum dimorphum BRAUN.}

(Taf. 1, Fig. 3 u. 9; Taf. 2, Fig. 12 u. 18.)

1850. Distomum dimorphum p. p. Diesing, Syst. helm., V. 1, p. 354. 1856. Distomum dimorphum p. p. Diesing, in: Denkschr. Akad. Wiss. Wien, math.-nat. Clo, V. 10 , p. 65, tab. 3, fig. 5, 6 .

1899. Clinostomum dimorphum BRAUN, in: Zool. Anz., V. 22, p. 490. 1900. Clinostomum dimorphum BraUn, in: Ctrbl. Bakt., V. 27, Abth. 1, p. 29.

Es scheint unverständlich, wie Diesing dazu kommen konnte, Trematoden von 60-100 mm Länge als weiter entwickelte Zustände von Formen anzusehen, die völlig entwickelt, aber nur 9-14 mm lang sind; zwischen beiden Formen sind grosse, schon für das unbewaftnete Auge sichtbare Unterschiede vorhanden, die nicht durch Zwischenglieder überbrückt werden; doch ist zu berücksichtigen, dass, wenn man, wie es Diesing that, die Organisation vernachlässigt, eine schein- 
bar zusammengehörige Reihe von den in Fischen encystirten Clinostomen über Clin. marginatum zu den noch grössern und einander in der Form ähnelnden Clin. detruncatum und sorbens führt - Arten, die Diesing von Clin. marginatum nicht sicher unterschieden hat; da sie nun in denselben resp. nahe verwandten Wirthen mit den riesig grossen Formen vorkommen und letztere doch auch manche Aehnlichkeiten mit ihnen aufweisen, so mag sich deshalb wohl Diesing über das Fehlen von Zwischengliedern hinweggesetzt und alle Exemplare für ein e Art gehalten haben.

Clin. dimorphum ist in der Wiener Sammlung nur in 8 Exemplaren vertreten; 6 fanden sich in Glas No. 832. X. 644, 2 andere in Glas No. 879. X. 853; beide stammen aus dem Oesophagus von Ardea cocoi.

Die Art ist die grösste unter den bisher bekannten Clinostom en und erreicht eine Länge, wie sie die Trematoden überhaupt nur selten aufweisen: 60-100 mm. Die grössere Vorderhälfte des Körpers ist lang gestreckt bandförmig bei einer Breite von 2-2,7 mm, nach hinten verbreitert sich der Körper allmählich bis auf $6 \mathrm{~mm}$, das letzte Ende ist wiederum verschmächtigt (Fig. 18). Die schmale Vorderhälfte ist auf der Ventralfläche ziemlich eben, auf der dorsalen stark gewölbt, wogegen das verbreiterte Hinterende auf der Dorsalfläche eine geringfügige Wölbung zeigt. Die hier unterschiedenen Körpertheile entsprechen jedoch nicht dem sonst bei Clinostomen unterscheidbaren Hals und Hinterleib; beide setzen sich auch deutlich bei Clin. dimorphum ab, doch liegt die Grenze weit vorn, dicht hinter dem grossen Bauchsaugnapf (Fig. 3).

Das Vorderende ist schräg nach der Ventralseite abgestutzt und trägt hier das grosse Mundfeld mit dem mehr oder weniger stark hervortretenden Mundkegel, auf dessen Ventralfläche die quer ovale Mundöffnung liegt; ihr Querdurchmesser beträgt beinahe $0,5 \mathrm{~mm}$. Entsprechend gross wird der Mundsaugnapf sein, ich habe ihn trotz aller Authellungsmittel nie so scharf begrenzt gesehen, dass ich ihn messen konnte.

Etwa $4 \mathrm{~mm}$ hinter der Mundöffnung bemerkt man die mehr dreieckige Eingangsöffnung in den 1,6 mm im Durchmesser zeigenden Bauchsaugnapf.

Die Darmschenkel, welche im Halstheil nur undeutlich hervortreten, umziehen im Bogen den Bauchsaugnapf, hinter ihm nähern sie sich wieder und lassen sich als gerade, einen bröckligen Inhalt führende Canäle, die im Ganzen parallel den Seitenrändern des Körpers 
nach hinten ziehen, bis ins Hinterende verfolgen; hier convergiren sie wiederum und enden blind in der Nähe des Excretionsporus. Aus der Form des geronnenen Inhalts lässt sich schliessen, dass sie wenigstens in dem verbreiterten Hinterende mit kleinen Blindsäckchen besetzt sind. Nach aussen von ihnen bemerkt man je einen hellen Gang, der dem Excretionsapparat angehört; auch sonst sind an einzelnen Stellen Theile der Excretionsgefässe als quer zum Seitenrand ziehende, an den Enden meist sich gabelnde Canälchen zu sehen, die dem oberflächlichen Gefässnetz angehören.

Das Genitaldrüsenfeld (Fig. 12), das schon auf den Abbildungen bei Diesing (l. c.) gezeichnet ist, liegt im Hinterende, ungefähr vom Hinterrand so weit entfernt, als es selbst lang ist ( $5-6 \mathrm{~mm})$. Die beiden Hoden sind vielfach gelappte, fast strahlige Körper, deren Vorder- wie Hinterrand gewöhnlich bogenförmig ausgeschnitten ist; in querer Richtung sind sie kaum mehr entwickelt als in der Längsrichtung. Der rechts gelegene Keimstock sieht von der Fläche halbmondförmig aus; seine Aussenfläche scheint etwas gelappt. Neben ihm in der Mittellinie liegt die hier mehr kuglige Schalendrüse, aus welcher ein sich links vom vordern Hoden nach vorn verlaufender, einige Schlingen bildender Canal entspringt, der Uterus; sein Durchmesser beträgt $0,33 \mathrm{~mm}$. Er mündet in das Hinterende des Uterussackes, der je nach seiner Füllung 7-12 mm lang ist; sein Hinterende ist bulbusartig erweitert und fast $2 \mathrm{~mm}$ breit. Die ihn füllenden Eier sind $0,125 \mathrm{~mm}$ lang und $0,06 \mathrm{~mm}$ breit.

In der Mittellinie dicht vor dem vordern Hoden liegt der Genitalporus; zu ihm geht vom Hinterende des Uterussackes ein kurzer Gang, während von rechts um den vordern Hoden herum der Cirrusbeutel herantritt, in welchem auch hier die Vesicula seminalis gelegen ist.

In der hintern Körperhälfte liegen die Dotterstöcke; fast genau in der Körpermitte trifft man die kleinen Follikel in je einer, den Darmschenkeln aufliegenden Längsreihen; etwas weiter nach hinten treten quere, etwas gebogen verlaufende Verbindungsreihen auf; zu ihnen gesellen sich andere nach den Seitenrändern sich erstreckende, und so entsteht ein ganz unregelmässiges Netzwerk, welches mit Ausnahme des Uterussackes und des Genitaldrüsenfeldes das ganze verbreiterte Hinterende einnimmt (Fig. 9 u. 12).

Aus dieser Beschreibung geht wohl mit Sicherheit die Berechtigung hervor, diese bis $100 \mathrm{~mm}$ lang werdende Form als besondere Art hinzustellen. 


\section{Clinostomum heluans BRAUN.}

(Taf. 2, Fig. 10.)

1899. Clinostomum heluans Braun, in: Zool. Anz., V. 22, p. 490.

1900. Clinostomum heluans Braun, in: Ctrbl. Bakt., V. 27, Abth. 1, p. 30 .

Im Sommer vorigen Jahres erhielt ich durch Herrn DE Muranda Ribeiro vom Nationalmuseum in Rio de Janeiro 2 Gläschen, welche je 2 Trematoden enthielten; die einen waren am 30. Mai 1898 in Ardea coerulea L., die andern am 1. Juni 1899 in Nycticorax gardeni Gu. und zwar beide in der Mundhöhle gesammelt worden. Alle 4 Exemplare gehören zu derselben Art, jedoch zu keiner der bisher beschriebenen; immerhin steht diese neue Art in nähern Beziehungen zu Clin. detruncatum.

Clin. heluans wird 10-12 $\mathrm{mm}$ lang und bis $2 \mathrm{~mm}$ breit; die Bauchfläche ist eben, bei gefülitem Uterussack springt dieser ventral als ein Längswulst etwas hervor; die Rückenfläche ist stark gewölbt. In der Höhe des Bauchsaugnapfes, etwa $1 \mathrm{~mm}$ vom Vorderende entfernt sondert sich der kurze Hals vom Hinterleibe durch eine Einziehung der Seitenränder deutlich ab. Das Mundfeld ist sehr klein und liegt terminal, ebenso der Mundsaugnapf, der $0,36 \mathrm{~mm}$ in der Längs- und $0,30 \mathrm{~mm}$ in der Querrichtung gross ist. Der Bauchsaugnapf ist etwa doppelt so gross $(0,62 \mathrm{~mm})$, sein Eingang rundlich oder dreieckig, seine Wand bis $0,19 \mathrm{~mm}$ dick.

Die D a r m s h en kel verlaufen ziemlich parallel den Seitenrändern bis ins Hinterende und sind vom Bauchsaugnapf an mit kurzen Blindsäckchen versehen. Der Oesophagus ist verhältnissmässig lang, dickwandig und verläuft vom Mundsaugnapf in der Medianebene in einem dorsalwärts gerichteten Bogen nach der Gabelstelle des Darms; daraus dürfte auf eine grosse Streckfähigkeit des Halses geschlossen werden können. Seine Einmündungsstelle in den Darm ist erweitert.

Ganz im Hinterende liegt das Genitaldrüsenfeld; von der Fläche gesehen sind die beiden Hoden etwa bohnenförmig mit etwas eingekerbtem Vorder- resp. Hinterrand; wie so häufig bei den Clinostomen ist auch hier der vordere Hoden der kleinere. Ueber die Grössenverhältnisse dieser Organe wie des Keimstockes bekommt man übrigens bei alleiniger Untersuchung von der Fläche bei Arten, welche stark gewölbt sind, ein falsches Bild: die Hoden erstrecken sich in dorsoventraler Richtung bis an die Muscularis heran; ihr Dorsoventraldurchmesser ist wohl ebenso gross wie der quere, der 0,7 resp. 
$0,9 \mathrm{~mm}$ beträgt. Von der Fläche gesehen erscheint der rechts zwischen den Hoden gelegene Keimstock kuglig, in Wirklichkeit stellt er ein dorsoventral stehendes Ovoid dar.

Anfangstheil des Uterus und Schalendrüse verhalten sich wie gewöhnlich; die Schalendrüsenzellen bilden insgesammt, wie eine Sagittalschnittserie ergiebt, einen Hohlmantel um das innere, gewundene Uterusende, der vorn wie hinten, dorsal wie ventral und auf der linken Seite geschlossen, dagegen rechts am Keimstock offen ist; zwischen die Uterusschlingen dringen die Schalendrüsenzellen weniger ein, doch fehlen sie nicht ganz. Der Keimleiter tritt von der medianen Fläche des Keimstocks ab, verbindet sich nach kurzem Verlauf mit dem ebenfalls kurzen, auf der Dorsalfläche rechts von der Mittelllinie mündenden LaURER'schen Canal, nimmt dann einen ganz kurzen, vom Dotterreservoir kommenden Gang auf und geht endlich in den sich vielfach windenden Anfangstheil des Uterus über. Ein Receptaculum seminis ist nicht vorhanden, jedoch fehlt Sperma in diesem Abschnitt des Uterus nicht.

Nachdem der Canal aus der Schalendrüse herausgetreten ist, wendet er sich am linken Seitenrande des vordern Hodens nach vorn, biegt dann hier medianwärts um und mündet vor dem vordern Hoden in den Uterussack ein. Je nach dem Füllungszustande stellt dieser entweder einen dickwandigen, aber kleinkalibrigen Schlauch dar, oder er ist nach allen Richtungen ausgedehnt und reicht dann seitlich bis an die Darmschenkel; seine vordere Grenze liegt vor der Körpermitte. Im gefüllten Zustande ist die ventrale und dorsale Wand des lang gestreckten Uterussackes einfach rinnenförmig, die seitlichen dagegen zeigen deutliche und ziemlich dicht stehende Ausbuchtungen, die mehr in dorsoventraler als in seitlicher Richtung entwickelt sind und daher nicht, wie bei Clin. detruncatum, als seitliche Sprossen erscheinen.

Das hintere Ende des Uterussackes zieht sich in einen schräg nach hinten gerichteten und die Bauchwand durchsetzenden Canal aus, der rechts von der Mittellinie nach aussen mündet; dicht dahinter, aber noch ein wenig mehr rechts ist der ausgestreckte Cirrus auf der Schnittserie zu sehen; aller Wahrscheinlichkeit nach besteht aber wohl auch bei Clin. heluans nur ein Genitalporus mit daran anschliessendem Genitalatrium, welches hier vorgestülpt ist.

Die Dotterstöcke stellen - von der Fläche gesehen - 2 seitlich gelegene, den ganzen Hinterleib einnehmende, breite Streifen dar, welche hinter den Hoden bogenförmig in einander übergehen; in 
Wirklichkeit erstrecken sich die Dotterstocksfollikel auch nach der Dorsalseite hin, so dass ein Querschnitt durch die Mitte des Hinterleibes ausser den quer getroffenen Darmschenkeln und dem Uterussack die Dotterstocksfollikel jederseits in bogenförmiger Anordnung, ventral, lateral und dorsal von den Darmschenkeln erkennen lassen würde; die Mitte der Rücken- wie Bauchfläche bleibt von ihnen frei und zwar die Rückenfläche in grösserer Ausdehnung.

Die Eier sind braun, oval, 0,11-0,14 mm lang, 0,069$0,078 \mathrm{~mm}$ breit.

\section{Clinostomum lambitans BRAUN.}

(Taf. 2, Fig. 16 u. 17.)

1899. Clinostomum lambitans Braun, in: Zool. Anz., V. 22, p. 490.

1900. Clinostomum lambitans Braun, in: Ctrbl. Bakt., V. 27, Abth. 1, p. 30.

Diese Art liegt in 2 Exemplaren aus dem Naturhistorischen Museum zu Hamburg vor; sie sind am 14. August 1894 von NepperSCHмIDT an der Semanabay (Westindien) im Schlunde eines reiherartigen Vogels gesammelt worden. Ich würde sie nicht beschreiben, wenn sich diese Art nicht leicht von allen bisher bekannten Clinostomen unterscheiden liesse, so dass ihr Wiedererkanntwerden mir sicher erscheint, obgleich ich nicht alle typischen Verhältnisse erkannt habe.

Clinostomum lambitans ist die kleinste Art, sie wird nur $2 \mathrm{~mm}$ lang und $0,6 \mathrm{~mm}$ breit; dem blossen Auge erscheinen die Thiere wie Dipteren-Eier, die übrigens nach meinen Erfahrungen in einzelnen Sammlungen als Helminthen aufbewahrt werden. Die Bauchfläche ist eben, die Rückenfläche ist ziemlich stark gewölbt. Der Hals setzt sich in der Höhe des Bauchsaugnapfes deutlich ab.

Auffallend gross im Verhältniss zur Kleinheit des Thieres ist das quer ovale Mundfeld $(0,57 \mathrm{~mm}$ im Querdurchmesser), der Mundsaugnapf hat einen Querdurchmesser von $0,16 \mathrm{~mm}$, der Bauchsaugnapf von $0,36-0,40 \mathrm{~mm}$.

Die Darmschenkel sind von der Gabelstelle an bis zum blinden Ende mit dicht stehenden Blindsäckchen versehen.

Die Genitalien verhalten sich ähnlich wie bei Clin. complanatum und $\mathrm{Cl}$. marginatum; der Genitalporus liegt rechts von der Mittellinie ungefähr in der Mitte zwischen dem Hinterrand des Bauchsaugnapfes und dem des Körpers; nur undeutlich sind in demselben Exemplar die beiden Hoden und der Keimstock erkennbar. Der Uterussack 
reicht bis zum Bauchsaugnapf; die dicht stehenden Dotterstocksfollikel nehmen die Seitenfelder sowie hinter den Hoden auch das Mittelfeld ein. Die Eier sind 0,073-0,091 $\mathrm{mm}$ lang und 0,052 $\mathrm{mm}$ breit.

Die Gattung Clinostomum enthält demnach bis jetzt 9 Arten, von denen 3 (Cl. heterostomum, complanatum und foliiforme) europäisch, die übrigen 6 amerikanisch sind; aus andern Erdtheilen ist bis jetzt nur die von v. Linstow als Dist. heterostomum beschriebene Form (aus Turkestan) bekannt, welche jedoch wahrscheinlich zu Cl. complanatum (Rud.) gehört. Ihre Wirthe sind: Ardea cinerea, A. purpurea, A. cocoi, A. coerulea, A. herodias, Ardea sp. VI (Brasilien), Ardea sp. (Westindien), Mycteria americana, Ciconia americana, Nycticorax griseus, N. gardeni, Tantalus loculator, Botaurus minor, und - wenn die Angabe von Stiles u. Hassall über die $\mathrm{Zu}$ gehörigkeit des Dist. aquilae LeIDy zu Clinostomum richtig ist ${ }^{1}$ ) auch Haliaëtus leucocephalus. Die Clinostomen scheinen durchweg den Vorderdarm ihrer Wirthe: Mundhöhle, Pharynx und Oesophagus zu bewohnen, Dist. aquilae dagegen ist in der Trachea gefunden worden, wohin es von der Mundhöhle aus leicht gelangen kann. Die Nahrung aller dieser Wirthe weist auf Fische als Vermittler der Infektion hin, aus denen in der That Jugendstadien der Clinostomen bekannt geworden sind.

\section{Jugendstadien der Clinostomen.}

Folgende Formen sind bis jetzt bekannt:

1) Clinostomum gracile LeIdy in: Proc. Acad. nat. Sc. Philadelphia V. 8 (1856), 1857, p. 45, gefunden im Darm von Esox sp. und encystirt bei Pomotis vulgaris. Das Vorkommen im Darm von Esox $s p$. muss als ein zufälliges bezeichnet werden, die Thiere befanden sich hier nach Allem, was wir wissen, sicher nicht an ihrem normalen Aufenthaltsorte. Beschreibung ganz unzureichend.

2) Distomum dimorphum forma primaria Dies., Syst. helm., V. 1, 1850 , p. 354 und in: Denkschr. Akad. Wiss. Wien, math.-nat. Cl., V. 10, 1856, p. 65, tab. 3, fig. 1-4; encystirt in Carapus brachy-

1) Diese Angelegenheit steht durchaus nicht so sicher, wie StrLEs u. Hassall anzunehmen scheinen; MacCaLLum, der ebenfalls den Typus von Dist. aquilae untersucht hat, stellt diese Form nicht zu Clinostomum, weil der Mundsaugnapf nicht von einem kragenartigen Ringwulst umgeben ist; über die Geschlechtsorgane erfahren wir allerdings von keinem Autor auch nur ein Wort. 
urus, Hydrolicus scomberoides, Geophagus pappatera, Chaetobranchus flavescens, Crenicichla johanna und C. lepidota; Mundfeld deutlich, Bauchsaugnapf mit dreieckigem Eingang, Genitaldrüsenfeld in der Mitte des Hinterleibes.

3) Distomum gracile Dies. = Clin. gracile LeIdy bei Wright in: Proc. Canad. Inst. (N. Ser.), V. 1, 1879, p. 9, encystirt bei Perca flavescens; $6,45 \mathrm{~mm}$ lang, grösste Breite $1,8 \mathrm{~mm}$; Mundsaugnapf 0,338 , Mundfeld 0,975 und Bauchsaugnapf $0,91 \mathrm{~mm}$ gross; keine Geschlechtsorgane. Der reife Zustand wird in einem Raubfisch oder Fische fressenden Vogel vermuthet, an Dist. heterostomum Wright (nec RUd.) resp. dessen Wirth ist hierbei nicht gedacht.

4) Distomum reticulatum Looss in: Z. wiss. Zool., V. 41, 1885, p. 427, Taf. 23, Fig. 20-29, encystirt bei einem mittelamerikanischen Siluroiden ${ }^{1}$ ); $9-11 \mathrm{~mm}$ lang, 2,5-3,5 mm breit, 0,75-1,0 mm dick. Mundsaugnapf fast kuglig $(0,195-0,238 \mathrm{~mm})$, Bauchsaugnapf etwa dreimal so gross, sein Eingang dreieckig. Pharynx fehlt, Darmschenkel bis ans Hinterende reichend, ohne Blindsäckchen. Genitaldrüsenfeld hinter der Mitte des Hinterleibes gelegen; Keimstock rechts; Cirrus- und Uterusmündung anscheinend links und ziemlich weit getrennt von einander, jeden Falls liegt der Cirrusbeutel zwischen den Hoden; der Uterussack erstreckt sich nach vorn bis zum Hinterrand des Bauchsaugnapfes, nach hinten über das Genitaldrüsenfeld hinaus.

5) Distomum galactosomum Leidy in: Proc. Acad. nat. Sc. Philadelphia (1888), 1889, p. 166; encystirt bei Labrax lineatus. Es ist bezeichnend, dass Leidy selbst die Zugehörigkeit dieser Form zu seinem 30 Jahre früher aufgestellten Genus Clinostomum nicht erkannt hat; erst MaCCaLlum stellt dies 1899 nach Untersuchung der Typen fest. 6-8, im ausgestreckten Zustande bis $12 \mathrm{~mm}$ lang, 2-2,5, am Hals $1,0 \mathrm{~mm}$ breit. Vorderende schräg abgestutzt, scheibenförmig mit vorspringendem Rand, 0,625 mm im Querdurchmesser (= Mundfeld); $1,4 \mathrm{~mm}$ vom Vorderende der 0,875 mm breite Bauchsaugnapf, dessen Mündung dreieckig ist. Darmschenkel bis ans Hinterende reichend, mit Blindsäckchen besetzt. Geschlechtsorgane nicht erkannt, scheinen in der Mitte des Hinterleibes zu liegen. Die milchweisse Farbe rührt von zahllosen Kalkkörperchen her, die mit Säure aufbrausen - von Kalkkörperchen spricht auch R. WRIGHT bei seiner Form.

1) Nicht in Silurus glanis, wie MacCallum meint. 
6) Distomum reticulatum PoIRIER in: Bull. Soc. philomath. Paris (sér. 7), V. 10, 1886, p. 39, tab. 3, fig. 8, encystirt bei Axinurus dugesi und für identisch mit der Looss'schen Form gehalten, was aber sicherlich nicht zutrifft; $5 \mathrm{~mm}$ lang, $2 \mathrm{~mm}$ breit, anscheinend bestachelt, was bisher von keiner Clinostomum-Art weder im erwachsenen noch im jugendlichen Zustande bekannt ist. Darmschenkel nur in der hintern Hälfte des Hinterleibes mit Blindsäckchen besetzt. Genitaldrüsenfeld in der Mitte des Hinterleibes; vorderer Hoden kleiner als der hintere; Keimstock kuglig, rechts zwischen blindem Ende des Cirrusbeutels und dem hintern Hoden; Cirrusbeutel rechts vor dem vordern Hoden, Genitalporus sicher ebenfalls vor diesem, anscheinend in der Mittellinie; Uterussack nur nach vorn sich erstreckend.

7) Distomum gracile Linton in: Proc. U. S. nation. Mus., Vol. 20, 1898 , p. 523; tab. 46, fig. 6-8, encystirt in Lepomis auritus, Eupomotis pallidus und Chaenobryttus gulosus. 4,5-7 mm lang, oblong-elliptisch, abgeflacht, Hals in der Höhe des Bauchsaugnapfes vom Hinterleib durch eine Einziehung an den Seitenrändern gesondert. Vorderende schräg abgestutzt, concav ausgehöhlt, hier der Mundsaugnapf $(0,27 \mathrm{~mm})$; Bauchsaugnapf mit dreieckiger Eingangsöffnung, etwa dreimal so gross; kein Oesophagus (??), Pharynx oval, Darmschenkel bis ins äusserste Hinterende reichend, vom Bauchsaugnapf an haustrirt. Genitaldrüsenfeld in der Mitte des Hinterleibes; Hoden quer gestreckt; Cirrusbeutel rechts vor dem vordern Hoden; Genitalporus in der Mittellinie vor den Hoden; Uterussack nach vorn bis zum Bauchsaugnapf reichend. Dotterstocksfollikel an den Seiten angedeutet.

8) Distomum gracile resp. Clinostomum heterostomum MacCallum in: Journ. Morph., V. 15, 1899, p. 704, tab. 39, fig. 7; dem Verf. lagen ausser den von WRIGHT aus Perca flavescens kurz beschriebenen Clinostomen noch encystirte Exemplare aus einem Frosch, einer Forelle und aus Ictalurus dugesi von Mexiko vor; die Formen aus der Forelle haben Stiles u. Hassall für Clinostomum heterostomum erklärt. Alle erscheinen dem Autor zu dieser selben Art zu gehören, von der der noch unentwickelte Genitalapparat auch abgebildet wird; die Exemplare aus der Forelle führten in der Haut feine Stacheln (cf. unter No. 6 PoIrier).

Aus dieser Zusammenstellung dürfte ebenfalls die Unrichtigkeit der Ansicht hervorgehen, dass alle diese Jugendstadien zu ein und derselben Species gehören; zu trennen ist vor allem Distomum reticulatum Looss (nec WRIGHT), weil bei ihr der Cirrusbeutel zwischen 
den Hoden liegt und der Uterussack sich bis hinter das Genitaldrüsenfeld fortsetzt; die einzige Clinostomum-Art, welche in Bezug auf die Lage des Cirrusbeutels mit der Looss'schen Form übereinstimmt, ist $\mathrm{Cl}$. sorbens; in anderen Punkten weicht sie aber ab, so dass eine Zusammenstellung beider zur Zeit wenigstens noch nicht möglich ist; immerhin sind beide Arten unter einander näher verwandt als mit andern Clinostomen.

Auszuscheiden sind ferner die ungenügend beschriebenen und nicht einmal bildlich dargestellten Formen, wie die LeIDY'sche und Wright'sche, ebenso auch diejenigen, von denen einfach angegeben wird, sie stimmen mit diesen resp. mit Clin. heterostowum überein; das muss nunmehr unter Berücksichtigung der hier gegebenen Beschreibungen erst festgestellt werden. So bleiben übrig Dist. reticulatum PoIrier 1886 (nec Looss, nec Wright), Dist. gracile Linton 1898 und die eine Form, deren Genitalapparat MacCallum 1899 abgebildet hat. Nach den vorliegenden Daten zu urtheilen, dürften die von POIRIER und Linton beschriebenen Formen identisch sein, trotzdem die erstere bestachelt sein soll; auch darf man sie mit grosser Wahrscheinlichkeit auf Clinostomum marginatum (RuD.) beziehen, da sie mit dieser Art am besten übereinstimmt. Dasselbe scheint mir auch für die von MACCALLum abgebildete Form zu gelten, die der Autor selbst zu Clin. heterostomum (nec RudolPHI), d.. h. zu Cl. marginatum (RUD.) stellt.

Was endlich die von Diesing kurz erwähnten und in einem Exemplar auch abgebildeten Jugendstadien von Clinostomen anlangt, so haben mir folgende zur Untersuchung vorgelegen:

1) No. 790. IX. 443 aus Gymnotus No. $70=$ Carapus brachyurus; zahlreiche gelbliche Exemplare, die theils frei, theils in Cysten im Abdomen liegen; gesammelt in Caiçara am 13. Oct. 1825.

2) No. 830. X. 608 aus Ghenza = Crenicichla johanna, zahlreiche Exemplare, die nach Diesing (1. c. 185̃6) unter der Haut am ganzen Körper, sowie an den Kiemen und Flossen einzeln in Kapseln sassen und von gelblichem Saft umgeben waren; gesammelt in Matogrosso am 14. August 1828. Das ebenda aus demselben Fisch erwähnte grosse, ockergelbe Distomum ist in dem Glase nicht mehr vorhanden.

3) No. 834. X. 610 aus Cichla $76=$ ? ein Exemplar; sass am Kiemendeckel und ist bei Diesing (1856) nicht angeführt.

4) No. 881. IX. 535 aus Salmo $74=$ Hydrolicus scomberoides; 
4 Exemplare, waren im Fleisch zwischen den Rippen encystirt und konnten schon von aussen gesehen werden; gesammelt am 22. Nov. 1826 in Matogrosso.

5) No. 917. X. 607 aus Salmo No. $74=$ Hydrolicus scomberoides; ein Exemplar frei im Darmcanale; gesammelt in Matogrosso am 18. August 1828 - auch das ist sicherlich ein zufälliger Befund.

Die übrigen an derselben Stelle angeführten, aus brasilianischen Fischen stammenden Clinostomen sind in der Wiener Sammlung nicht mehr vorhanden.

Die vorhandenen Exemplare stimmen alle unter einander überein; ihre Längen schwanken zwischen 4 und $6 \mathrm{~mm}$; der Körper ist flach, nur andeutungsweise auf dem Rücken gewölbt; der Hals setzt sich meist deutlich in der Höhe des kräftigen Bauchsaugnapfes ab und entspricht - je nach der Streckung des Körpers - einem Drittel bis einem Viertel der ganzen Körperlänge. Das Mundfeld ist entweder vorgewölbt oder eingezogen, sein Querdurchmesser beträgt 0,8 $-1,13 \mathrm{~mm}$; der Mundkegel tritt nur selten hervor, wohl aber meist der Mundsaugnapf, der bald etwas mehr in die Quere oder mehr in die Länge gestreckt oder rundlich ist; die Durchmesser schwanken zwischen 0,21-0,3 mm, die des Bauchsaugnapfes, der stets eine dreieckige Eingangsöffnung besitzt, zwischen 0,6 und $0,9 \mathrm{~mm}$.

Das Genitaldrüsenfeld ist in den kleinen Exemplaren oft nur angedeutet, in den grössern sind wenigstens die Hoden, doch gelegentlich auch der Cirrusbeutel, Uterus und Uterussack, sowie der Keimstock und die Schalendrüse zu sehen; am seltensten habe ich die Dotterstöcke erkannt, dagegen sind namentlich bei kleinern Exemplaren Theile des oberflächlichen Gefässnetzes, gelegentlich auch je ein Längsstamm an den Seiten deutlicher zu sehen; in wenigen erkannte ich auch die schon von andern Autoren erwähnten Kalkkörperchen ähnlichen Bildungen.

Das Genitaldrüsenfeld liegt gewöhnlich vor der Mitte des Hinterleibes; die Hoden sind meist schmal und quer gestreckt, mitunter auch mehr dreieckig; ihre einander zugekehrten Ränder sind gewöhnlich etwas concav, die andern gezackt oder eingekerbt. Die Geschlechtsöffnung habe ich nur ausnahmsweise sehen können, sie lag dann vor dem vordern Hoden in der Mittellinie.

Da nun nicht wenige Exemplare, namentlich aus Glas 830, Exemplaren aus der Rachenhöhle von Ardea cocoi (Glas No. 831) zum Verwechseln gleichen (Fig. 19) und ich die letztern zu Clinostomum marginatum (RUD.) gestellt habe, so bin ich der Meinung, dass alle 
mir vorliegenden Jugendstadien von Clinostomen aus brasilianischen Knochenfischen zu derselben Art gehören. Diese würde dann die häufigste Art darstellen und über Süd- und Nordamerika verbreitet sein. In Europa sind bisher Clinostomenlarven in Fischen überhaupt noch nicht gefunden worden, so dass die Vermuthung wohl gerechtfertigt ist, die Infection der Clinostomen beherbergenden europäischen Vögel (Ardea, Nycticorax) finde in Süd-Europa oder Nord-Afrika statt.

\section{Erklärung der Abbildungen.}

Alle Abbildungen (excl. Fig. 14) stellen auf dem Rücken liegende Exemplare dar.

T a fel 1.

Fig. 1. Clinostomum heterostomum (RUd.), $15: 1$. Aus Ardea purpurea, Genua, 13./5. 1887. Mk Mundkegel, Bl Darmblindschläuche, $K$ Keimstock.

Fig. 2. Dasselbe, Hinterleib mit Darmschenkeln und Genitalien. Bsg Bauchsaugnapf, Dsch Darmschenkel, G.p Genitalporus, Hh hinterer Hoden, $H v$ vorderer Hoden, $K$ Keimstock.

Fig. 3. Clinostomum dimorphum BRAUn, aus Ardea cocoi (Wiener Sammlung, Glas No. 832). Vorderende bei auffallendem Licht. 12:1.

Fig. 4. Clinostomum marginatum (RUd.), Typus, aus Ardea sp. VI. (Berliner Sammlung, No. 1493). 20:1.

Fig. 5. Clinostomum foliiforme BRAUn, aus Ardea purpurea (Turiner Sammlung, CN. 361). 30:1.

Fig. 6. Clinostomum complanatum (Rud.), Typus, aus Ardea cinerea Berliner Sammlung, No. 1460). 20:1.

Fig. 7. Clinostomum complanatum (RUD.), aus Ardea cinerea (Genua, 10./4. 1886), stark gestreckt. $15: 1$. G.p Genitalporus.

Fig. 8. Clinostomum marginatum (Rud.), aus Ardea cocoi (Wiener Sammlung: 831. X. 644). $20: 1$.

Fig. 9. Clinostomam dimorphum Braun, aus Ardea cocoi (Wiener Sammlung: 879). 9:1. Mittlerer Theil des Körpers mit Dotterstock und Vorderende des Uterussackes.

\section{Tafel 2 .}

Fig. 10. Clinostomum heluans BRAUn, aus Ardea coerulea, Rio de Janeiro, 8./7. 1899 (de Muranda Ribeiro leg.) $20: 1$. 
Fig. 11. Clinostomum detruncatum Braun, aus Mycteria americana (Wiener Sammlung No. 750). 15 : 1.

Fig. 12. Clinostomum dimorphum Braun, aus Ardea cocoi (Wiener Sammlung, Glas No. 832); Hinterende mit dem Genitaldrüsenfeld. 9: 1.

Fig. 13. Clinostomum sorbens BRAUn, aus Tantalus loculator (Wiener Sammlung, No. 878). 20: 1.

Fig. 14. Dasselbe Exemplar, auf dem Bauche liegend. $20: 1$.

Fig. 15. Clinostomum sorbens, ebendaher, bei auffallendem Licht gez. $12: 1$.

Fig. 16. Clinostomum lambitans Braun, aus Ardea sp. (Hamburger Sammlung, No. 17900). 38: 1 .

Fig. 17. Clinostomum lambitans BRAun, ebendaher, 38:1.

Fig. 18. Clinostomum dimorphum Braun, aus Ardea cocoi (Wiener Sammlung, No. 832). Nat. Grösse.

Fig. 19. Clinostomum marginatum (RUD.), unausgewachsenes Exemplar aus Ardea cocoi (Wiener Sammlung, 831. X. 644). 20 : 1. Oberflächliches Netz der Excretionsgefässe deutlich.

Fig. 20. Clinostomum marginatum (RUD.), ausgewachsenes Exemplar, ebendaher. 20:1. 


\section{$2 \mathrm{BHL}$ Biodiversity Heritage Library}

1900. "Die Arten der Gattung Clinostomum Leidy." Zoologische Jahrbücher 14, 1-48. https://doi.org/10.5962/bhl.part.11527.

View This Item Online: https://www.biodiversitylibrary.org/item/38157

DOI: https://doi.org/10.5962/bhl.part.11527

Permalink: https://www.biodiversitylibrary.org/partpdf/11527

\section{Holding Institution}

MBLWHOI Library

\section{Sponsored by}

MBLWHOI Library

\section{Copyright \& Reuse}

Copyright Status: NOT_IN_COPYRIGHT

This document was created from content at the Biodiversity Heritage Library, the world's largest open access digital library for biodiversity literature and archives. Visit BHL at https://www.biodiversitylibrary.org. 